\title{
Electrochemically Crafted Monodispersed y-Cu5Zn8 Bimetallic Nanoalloy: Characterization and Its Synergistic Effect on Photocatalysis and Antibacterial Efficacy
}

\section{R. Singaravelan}

Department of Chemistry, Saveetha School of engineering, Thandalam

\section{Shanmugam}

Department of Civil Engineering, Yeungnam University

\section{A. Abdul Salam}

B. S. Abdur Rahman Crescent Institute of Science and Technology

P. Vasanthi

B. S. Abdur Rahman Crescent Institute of Science and Technology

\section{Magesh}

Department of Material Science, National Taiwan University

D. Mani

Department of Civil Engineering, Yeungnam University

Young-Ho Ahn ( $D$ yhahn@ynu.ac.kr)

Department of Civil Engineering, Yeungnam University https://orcid.org/0000-0002-8301-5886

\section{Research Article}

Keywords: $y$-CuZn nano alloy, the electrochemical method, photocatalytic activity, antibacterial efficacy.

Posted Date: March 1st, 2021

DOI: https://doi.org/10.21203/rs.3.rs-167899/v1

License: (c) (i) This work is licensed under a Creative Commons Attribution 4.0 International License. Read Full License 


\title{
Electrochemically crafted monodispersed $\gamma-\mathrm{Cu}_{5} \mathrm{Zn}_{8}$ bimetallic nanoalloy: characterization and its synergistic effect on photocatalysis and antibacterial efficacy
}

\author{
R. Singaravelan ${ }^{\mathbf{a} 1}$, M. Shanmugam ${ }^{\mathbf{a} 4}$ A. Abdul Salam ${ }^{2}$, P. Vasanthi ${ }^{2}$, M. Magesh ${ }^{3}$, D. Mani ${ }^{* 4}$, \\ Young-Ho Ahn $4 *$ \\ ${ }^{1}$ Department of Chemistry, Saveetha School of engineering, Thandalam, Chennai, India \\ 2, B. S. Abdur Rahman Crescent Institute of Science and Technology, Chennai, India \\ ${ }^{3}$ Department of Material Science, National Taiwan University, Taiwan \\ ${ }^{4}$ Department of Civil Engineering, Yeungnam University, Gyeongsan, South Korea \\ ${ }^{a}$ These authors contributed equally to this work \\ *Corresponding authors: E-mail: Young-Ho Ahn: yhahn@ynu.ac.kr \\ Durai Mani: manivivekajan5@gmail.com
}

\begin{abstract}
:
The present work reports the investigation of monodispersed nano brass prepared by the facile electrochemical technique. The influence of reaction conditions on the surface morphology and size of the bimetallic nano alloy were investigated through high resolution scanning electron microscopy (HR-SEM) and powder X-ray diffraction (PXRD) technique. From the powder XRD analysis the nano alloy was identified as a $\gamma-\mathrm{Cu}_{5} \mathrm{Zn}_{8}$ phase. The detailed investigation was carried out using transition electron microscope (TEM) for the textural features and $\gamma$-CuZn phase stoichiometry was revealed by X-ray photoelectron spectroscopy (XPS) and energy dispersive Xray spectroscopy (EDAX) analyses. Optical responses were analysed using diffuse reflectance spectroscopy (DRS). The energy band gap obtained from the optical spectrum revealed it can be a good catalyst. Rate of photocatalytic reaction and mechanism of degradation were discussed in detail. Most importantly, electrodeposited $\gamma$-CuZn nano alloy reveals superior nano-catalytic
\end{abstract}


activity on methyl orange dye degradation in the shorter irradiation time. Furthermore, the antimicrobial efficacy of the $\gamma$-CuZn nano alloy was examined using gram positive and gram negative bacterial strains.

Keywords: $\gamma$-CuZn nano alloy, the electrochemical method, photocatalytic activity, antibacterial efficacy.

\section{Introduction}

Bimetallic nanostructures have fascinated the considerable research attention for their significant applications in science and technology [1]. It has also been demonstrated that these bimetallic nano alloys showed a direct influence on their size, shape etc [2]. In general nano alloys are composed of two or more different metals having a definite relation for alloying patterns or interaction sequences and geometry for structural design which perform specific functions $[3,4]$. Reinforced bimetallic nano alloys exhibit a considerable difference in their properties compared to monometallic analogous, particularly as an effective catalyst [5]. The aim of this investigation is to describe the simple as well as convenient method to fabricate nano-alloys with metals having dissimilar properties [6]. Electrodeposition was selected for the synthesis of CuZn bimetallic NPs because of its cost-effectiveness, simplicity, and ease of availability. Using this technique one can easily get the nano-alloy with desired structural morphologies through optimizing the reaction conditions for instance the precursor concentration, applied current, and $\mathrm{pH}$ etc [7]. The major challenge towards the electrodeposition of nano-brass involves the simultaneous deposition of both the ions under the optimal conditions (cathode over-potential) [8]. The problem which remains unexplored in this work is that the reaction parameter responsible for the phase determination. However, the change in electrolyte concentration results in cluster formation [9]. 
The effect of applied current is the primary factor which determines the nature and size of the nano-alloy. Secondly the electrolyte concentration of the corresponding metal ions from which the $\gamma$ - phase of nano-alloy is determined [10]. The preparation methods associated with chemical means, electrochemical route for the synthesis of alloy/bimetallic nanostructures have extended substantial research attention in recent times owing to its practical benefits, for instance easy, fast preparation, and no toxic reagents are used. Controlled synthesis of shape, size, and composition in addition to the thickness of bimetallic nanoparticles can be achieved through varying electrodeposition parameters $[11,12]$.

Organic effluents from dye and food industries represent major contamination to the water resources. A major portion of commercial dyes are released out untreated as leachate that leads to environmental pollution to the large extent. Methyl orange is a cationic diazo compound from the azo dye family. It is commonly used in food industries as a colouring agent and in textile industry for dyeing the synthetic materials as it does not work on cotton. It is mutagenic in living organisms and can cause permanent damage to the lives. It normally causes skin and eye irritation, and it has hazard in the solid form that can damage the lung tissue which increases the heart rate as a consequence.

With the never-ending environmental alarms, the quest for active catalyst having good response to the visible light photo catalysis for effective environmental remediation is still continuing and materialized as an important insertion area of research [13, 14]. Environmental friendly photocatalytic materials are preferred as an effective photocatalysts for dye degradation. Semiconductor materials were highly focused for the photocatalytic applications for their unique features $[15,16]$. Though these semiconductor materials fails to be an active material in absorption of visible light and are fast in recombination of photo generated positive 
hole and electron pair. Hence, hybrid nanomaterials with hierarchical structure with enhanced photo response offers very good photocatalytic activity [17, 18].

Owing to enhanced catalytic activity nano alloys are gaining more importance compared to their metallic counterparts [19-26]. Owing to the unique properties of nano alloys, they have been used as the functional materials in sensors, optics, electronic devices, and catalysts. The nature of the bimetallic NPs can be a core-shell structure otherwise in alloy form based on the preparation conditions. Amongst a number of bimetallic NPs, remarkable attention has been devoted to bimetallic nanostructures of Copper for outstanding characteristics [21-23]. Few reports have been available for the preparation of $\mathrm{Cu} / \mathrm{Pd}$ nano alloy using various techniques [24-27]. On the other hand, as far as our knowledge is concerned, there is no literature on the electrochemical synthesis of $\gamma-\mathrm{CuZn}$ bimetallic nano alloy with its photocatalytic and antibacterial activities. $\mathrm{Cu} / \mathrm{Zn}$ nano alloys are cost-effective compared to Ag NPs and Au NPs. Hence, we prepared stable $\gamma$ CuZn bimetallic nano alloy without a chemical reducing agent by the facile electrochemical method. The photocatalytic activity of $\gamma$-CuZn nano alloy was studied using methyl orange as a model dye.

Further, the well-diffusion assay (microdilution process) technique was used to evaluate the antimicrobial activity of $\gamma$-CuZn nano alloy on gram-positive microorganism bacillus subtilis and staphylococcus aureus (MRSA) and gram-negative pathogens Klebsiella pneumoniae and E. coli. 


\section{MATERIALS AND METHODS}

\subsection{Materials}

Analytical grade sulphate salts of copper and zinc $\left(\mathrm{CuSO}_{4} \cdot 5 \mathrm{H}_{2} \mathrm{O}\right)$ and $\left(\mathrm{ZnSO}_{4} \cdot 7 \mathrm{H}_{2} \mathrm{O}\right)$, were used as the precursors for the bimetallic CuZn nano alloy. Sodium sulphate $\left(\mathrm{Na}_{2} \mathrm{SO}_{4}\right)$ was used in required proportion as a supporting electrolyte and the electrolyte $\mathrm{pH}$ is controlled by the reducing agent Hydrazine sulphate monohydrate $\left(\mathrm{N}_{2} \mathrm{H}_{6} \mathrm{SO}_{4} \cdot \mathrm{H}_{2} \mathrm{O}\right)$, conductivity water (Merck) was used to prepare the electrolyte. The electrolyte was pre-treated with $\mathrm{N}_{2} \mathrm{H}_{6} \mathrm{SO}_{4}$ for the inert atmosphere in the electrolyte. Carbon electrodes of $75 \mathrm{~mm}$ long with $12 \mathrm{~mm}$ diameter were used as working and counter electrodes. The carbon rods were degreased with acetone followed by washed with, deionized water before the electrolysis. The electrodeposition of CuZn bimetallic NPs was carried out at a constant current of $15 \mathrm{~mA}$ per second using a regulated power supply unit in the acidic condition.

\subsection{Synthesis of CuZn bimetallic nano alloy}

Electrodeposition was selected for the synthesis of CuZn bimetallic NPs because of its cost-effectiveness, simplicity, and ease of availability. Copper is more electropositive $(0.34 \mathrm{eV})$ compared to zinc $(-0.76 \mathrm{eV})$, consequently, copper will preferentially be deposited. However, the bimetallic NPs obtained contained predominantly Zn.

At lower current densities, copper ions have sufficient time to diffuse in from bulk solution and replace the ions being deposited. At a higher applied current, the molarity difference between copper and zinc becomes more significant at the working electrode. Copper is deposited at its limiting applied current, but zinc deposition is restricted. Copper ions slowly diffuse in from the bulk solution while sufficient $\mathrm{Zn}$ ions are available for deposition to occur. Zinc is preferentially deposited as the copper ion concentration is considerably reduced in the electrolyte. CuZn 
bimetallic deposited at the high-applied current was found to be rich in zinc while the deposition at the low-applied current was found to be rich in copper and the interface area between the two electrodes showed a smooth variation in composition. The formation of CuZn alloy is depicted in the following scheme.

At low applied current,

$$
\mathrm{Zn}^{2+}+\mathrm{Cu}^{2+}+2 \mathrm{e}^{-} \rightarrow \mathrm{Zn}_{\mathrm{aq}}^{2+}+\mathrm{Cu}^{0}
$$

At higher applied current,

$$
\begin{gathered}
\mathrm{Zn}^{2+}+\mathrm{Cu}^{2+} \stackrel{\mathrm{e}^{-}}{\longrightarrow} \mathrm{Zn}^{+}+\mathrm{Cu}^{0} \\
\mathrm{Zn}^{+}+\mathrm{Cu}^{0} \stackrel{\mathrm{e}^{-}}{\longrightarrow} \mathrm{ZnCu}
\end{gathered}
$$

\subsection{Instrumentation and Analysis}

Various physio-chemical characterization techniques have been used to characterize as synthesized nano-catalyst. In order to delineate the phase structure of the CuZn bimetallic NPs; Xray Diffractometer (XRD) Siemens-D-500 with $\mathrm{Ni}$-filtered $\mathrm{Cu} \mathrm{K} \alpha$ radiation operated at $30 \mathrm{kV}$ with $10 \mathrm{~mA}$ emission current. Information on diffractions were picked up over the range of $2 \theta$ from $10^{\circ}$ to $80^{\circ} \mathrm{C}$ with a scan rate of $7 \%$ min. High Resolution - Scanning Electron Microscope FEI Quanta FEG 200 SEM with EXS was used to observe the surface morphology and the stoichiometry the nano alloy. Diffuse reflectance spectrometer Shimadzu 2100 with BaSO4 as the reference material was used for optical characterisations. Functional group analysis was carried out with Fourier Transform Infra-Red (Perkin Elmer RX1 instrument) spectrophotometer. Transmission Electron Microscopic (TEM) studies were recorded by using JEOL 3010 UHER pole piece model instrument.

\subsection{Photocatalysis experiment}

The photo degradation process was optimized with $0.01 \mathrm{~g} / \mathrm{L}$ of nano-catalyst and $50 \mathrm{mg} / \mathrm{L}$ of dye concentration. Before the irradiation process, the dye solution is mixed with the nano- 
catalyst for $30 \mathrm{~min}$ in the dark in order to achieve the equilibrium and subsequently irradiated. A small portion of the aliquot was withdrawn and analysed for its absorbance measurements. The efficiency of degradation percentage is calculated using the formula.

$$
\% \text { of degradation }=\frac{C_{0}-C_{t}}{C_{0}} \times 100
$$

\section{RESULTS AND DISCUSSION}

\subsection{Optimization of reaction conditions for the electrodeposition of $\mathrm{CuZn}$ bimetallic}

High resolution scanning electron microscopy (HR-SEM) characterization was performed to identify the optimum condition for the electrodeposition of $\mathrm{CuZn}$ bimetallic nanoparticles. It is observed that the HR-SEM micrographs of the bimetallic nanoparticles have an excellent uniformity and well connected crystals in a coral like structure. It is observed that the HR-SEM micrographs of bimetallic nanoparticles have excellent uniformity and well connected crystal grains in a coral like structures. Figure $1 \mathrm{a} \& \mathrm{~b}$ display the images of bimetallic nanoparticles with 2:1 and 1:1 proportion of $\mathrm{Cu}-\mathrm{Zn}$ at $\mathrm{pH} 4.5$ in the applied current of $20 \mathrm{~mA}$. With increase in the concentration of electrolyte at $20 \mathrm{~mA}$ current with $\mathrm{pH} 5.5$ degree of aggregation increases with deformation in coral structure as shown in the fig. $1 \mathrm{c}$. and it is also observed that the coral structure was retained when the $\mathrm{pH}$ is reduced to 4 , as demonstrated in the fig. $1 \mathrm{~d}$. figure $\mathrm{c} \& \mathrm{~d}$ also reveals the decreased surface are of the particles leading to more aggregation due to change in reaction condition. Decrease in aggregation of nanoparticles with distorted cubical arrangement of atoms is clearly is seen in the fig e \& $\mathrm{f}$ as the electrolyte concentration, $\mathrm{pH}$ and applied current are optimized to $1: 1(10 \mathrm{mM}), 3.5$ and $10 \mathrm{~mA}$ respectively and the optimized result is shown in fig. $\mathrm{f}$. Unlike pure copper and pure zinc, the bimetallic nano alloy is easily clustered together, even at 
very low current (electrostatic condition). Hence, the method of preparation is suitable for the bimetallic nanoparticles fabrication.

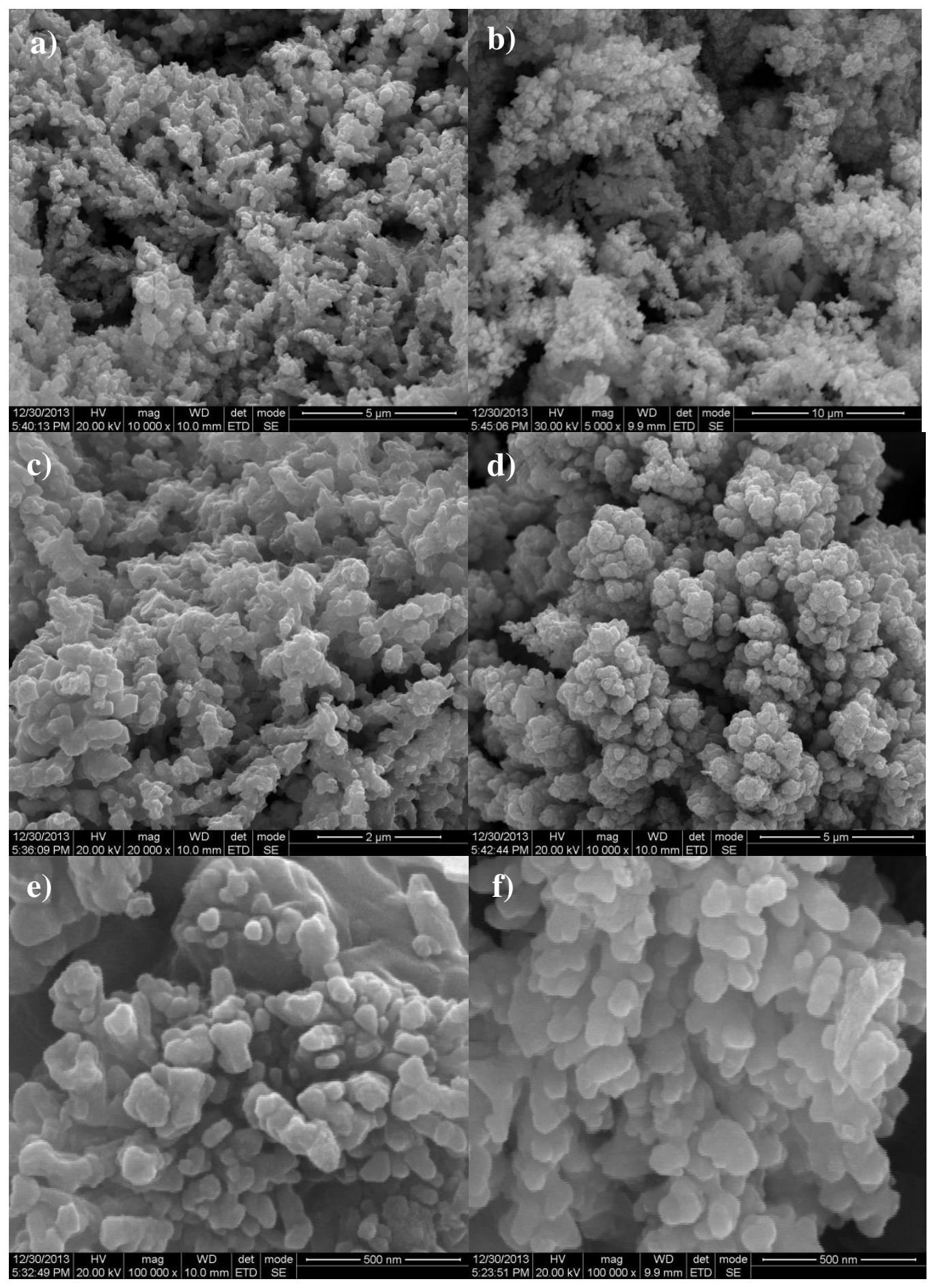


Figure 1. HR-SEM micrographs of $\mathrm{CuZn}$ bimetallic nanoparticles obtained at different reaction parameters (a-b) at a pH 4.5 with 0.02:0.01 mM concentration of $\mathrm{Cu}: \mathrm{Zn}$ at 20mA applied current, c) at a pH 5.5 with $0.02: 0.02 \mathrm{mM}$ conc. at $20 \mathrm{~mA}$, d) at a $\mathrm{pH} 4$ with $0.02: 0.02 \mathrm{mM}$ at $20 \mathrm{~mA}$, e) at a pH 3.5 with $0.01: 0.01 \mathrm{mM}$ at $20 \mathrm{~mA}$, f) at a $\mathrm{pH} 3.5$ with $0.01: 0.01 \mathrm{mM}$ at $10 \mathrm{~mA}$ (optimized condition)

\subsection{Structural confirmation}

Figure 2 (a-c) show the diffractograms of CuZn nanocatalyst prepared from various reaction parameters. The X-ray diffractogram results point out that the predominant diffraction signals for pure $\mathrm{Cu}$ and pure $\mathrm{Zn}$ were not recorded confirming the pattern obtained belong to the $\gamma$-CuZn phase nano alloy. The characteristic peaks of $\mathrm{CuZn}$ observed in the samples at $36.4^{\circ}, 38.6^{\circ}$, $43.3^{\circ}, 50.4^{\circ}, 53.66^{\circ}, 61.4^{\circ}$ and $74.2^{\circ}$ are attributed to the (222), (321), (330), (422), (510), (600) and (444) planes, which can be indexed to a body-centred cubic phase of $\gamma$-CuZn with lattice constant $\mathrm{a}=8.860 \AA$, (JCPDS 25-1228). In all XRD patterns, an appreciable peak observed at $2 \theta$ angle $43.2^{\circ}$, is associated with the (330) plane fighting fit pointing to the extraordinary crystallinity of $\gamma$-CuZn bimetallic nano alloy. The XRD results show that the change in the reaction parameters did not change the structure of the alloy obtained instead the peaks observable that centred at $35.05^{\circ}$ and $37.96^{\circ}$, which are attributed to the (222) \& (321) plane of $\mathrm{CuZn}$ showed shifting of peak to lower angle as a consequence of increasing $\mathrm{Cu}$ content. In addition, the above shift in peak positions are not because of the true expansion of the unit cell since the peaks at higher angels would have a larger shift compared to those at lower theta values. For the cubic materials, stretching of the cell might result with shift in the reflections towards the subordinate angle, although the extent of the shift would not be constant throughout the pattern. 
High crystallinity is an advantageous feature that determines the photocatalytic efficacy of the bimetallic. The defects are performing the role of entrapping/recombination centres for photogenerated electron-hole pair, causing decrease in photocatalytic efficiency. Larger particles will possess higher recombination probability, which is an undesirable quality for photocatalytic applications. Hence, higher the crystallinity smaller is the defects in it. These findings clearly indicate that the body centre cubic (BCC) $\mathrm{CuZn}$ bimetallic nano alloy was synthesized from the electrodeposition technique. Especially the X-ray diffraction analysis show that the sample prepared in the optimized condition (in accordance with stoichiometry ratio) exhibited good crystalline nature.

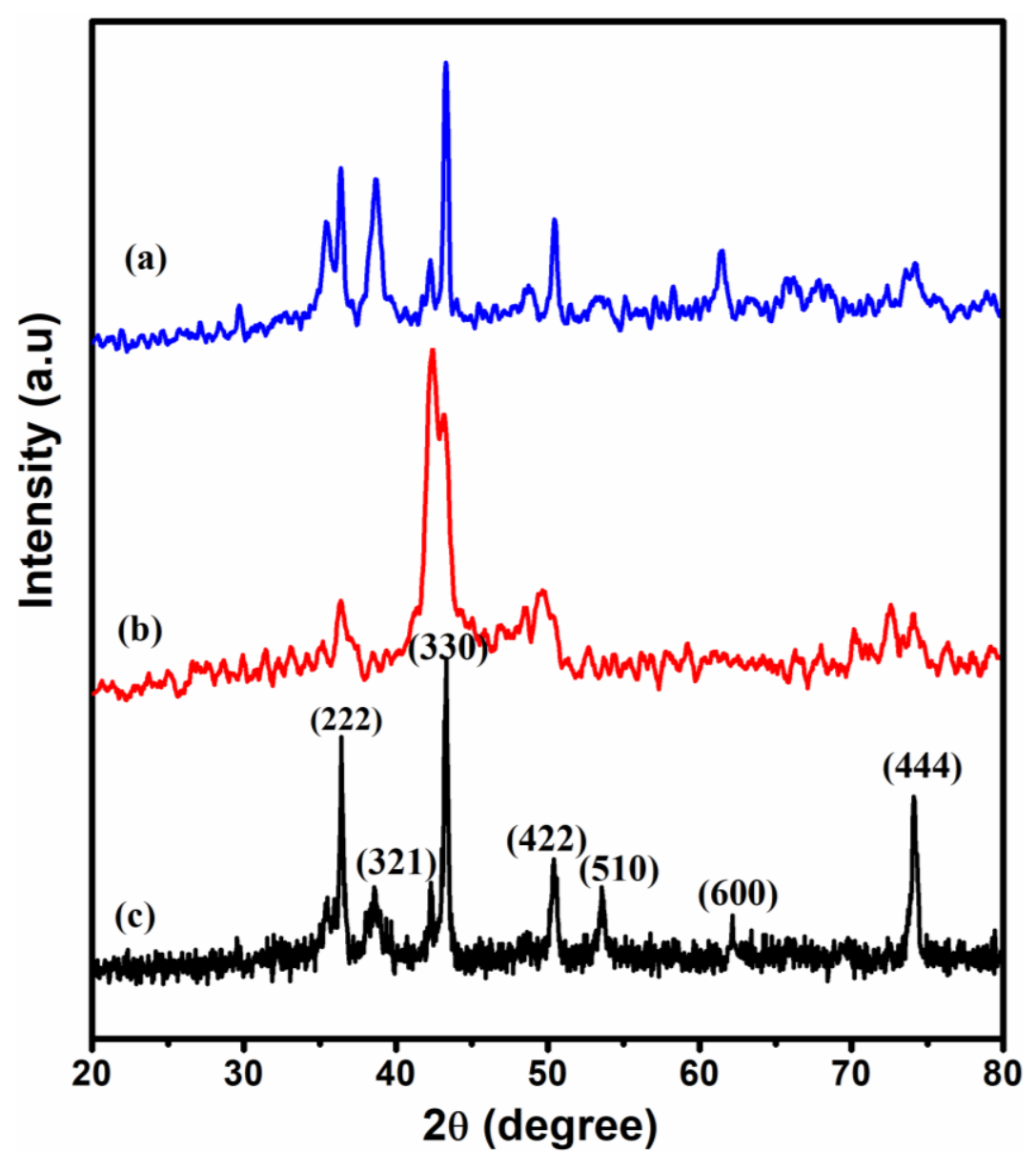


Figure 2. XRD patterns of $\mathrm{CuZn}$ bimetallic nano alloy electrodeposited on to the graphite electrode at different reaction conditions. A) at a $\mathrm{pH} 4.5$ with $0.02: 0.01 \mathrm{mM}$ concentration of $\mathrm{Cu}: \mathrm{Zn}$ at $20 \mathrm{~mA}$ applied current, B) at a pH 5.5 with 0.02:0.02 mM conc. at $20 \mathrm{~mA}$, e) at a pH 3.5 with 0.01:0.01 $\mathrm{mM}$ at $20 \mathrm{~mA}, \mathrm{C}$ ) optimized condition - at a pH 3.5 with 0.01:0.01 mM at $10 \mathrm{~mA}$

The average particle size and a shift in peak positions due to uniform strain in the nanocrystals were estimated using Debye-Scherrer formula and Williamson-Hall equation respectively. The average crystallite size of $\gamma-\mathrm{CuZn}$ alloy was estimated through the line broadening analysis by Debye-Scherrer equation [28]:

$$
\mathrm{D}=\frac{\mathrm{K} \lambda}{\beta_{\mathrm{hkl}} \operatorname{Cos} \theta}
$$

Where, $D$ is the crystalline size (nm), $K$ the shape factor $(0.9), \theta$ is the diffraction angle, $\beta$ the full width half maximum $(F W H M)$ of X-ray diffraction peak (rad.), $\lambda$ is the wavelength of $\mathrm{CuK} \alpha$ radiation in $\mathrm{nm}$. The average crystallite size of $\mathrm{CuZn}$ synthesized from different reaction conditions was found to be $16.21 \mathrm{~nm}, 5.56 \mathrm{~nm}$, and $10 \mathrm{~nm}$. The lattice parameter calculated was also in agreement with the reported values. A line broadening in all the diffraction reflections indicates the nanoscale properties of the CuZn bimetallic alloy.

The mean strain and size of a powder can be calculated from the diffraction pattern even when both are present simultaneously. The estimated formulae for strain $(\tan \theta)$ and size $(1 / \cos \theta)$ broadening vary in their own way with respect to Bragg's angle. The combined effect of both size and strain are would be determined by convolution (either simple sum or the sum of squares) in case both influences are present using the simplified Williamson Hall equation.

Using WH equation of these two, 


$$
\beta_{\text {total }}=\beta_{e}+\beta_{D}=C \varepsilon \tan \theta+\frac{K \lambda}{\mathrm{DCos} \theta}
$$

Multiplying by $\cos \theta$,

$$
\beta_{t o t a l} \cos \theta=C \varepsilon \sin \theta+\frac{K \lambda}{D}
$$

By plotting (Williamson Hall plot) $\beta_{\text {total }} \cos \theta$ versus $C \sin \theta$ size component can be obtained from the intercept whereas the strain factor is acquired from the slope. When the points in the W-H plot are strewn, that is $\beta_{\mathrm{hkl}} \cos \theta$ is not a repetitious function of $4 \sin \theta \operatorname{such}$ broadening in the diffraction profile was essentially anisotropic, which confirmed the CuZn bimetallic obtained are in cubic crystalline nature. The uniform deformation models for the $\mathrm{CuZn}$ are presented in fig. 3 (a-c). Table 1 summarizes the geometric parameters of the CuZn bimetallic synthesized from various reaction conditions.

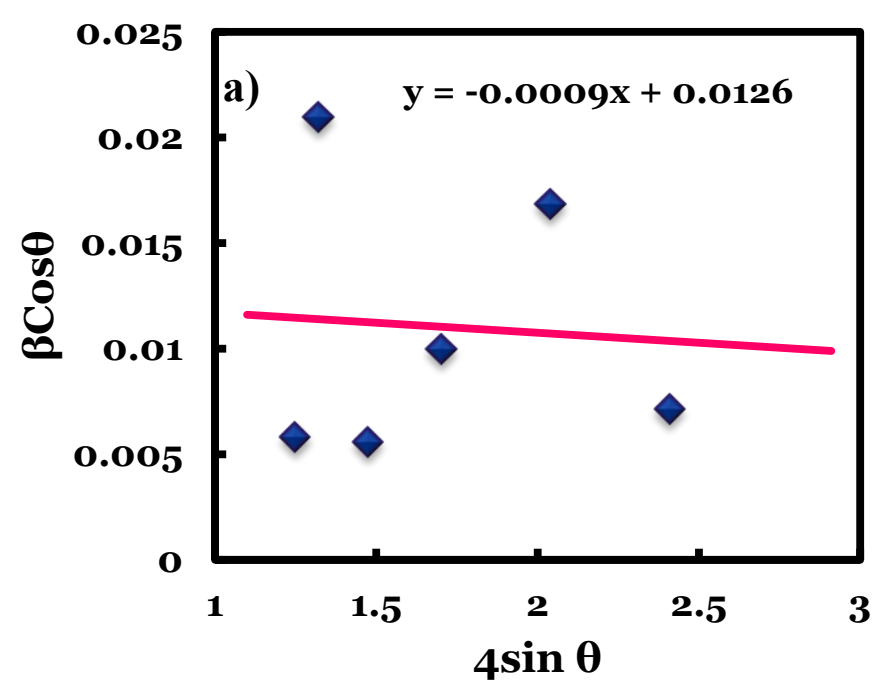

Slope $=-\mathbf{0 . 0 0 0 9}$ Particle size $=\mathbf{1 1 . 0 1 2 8}$ nm

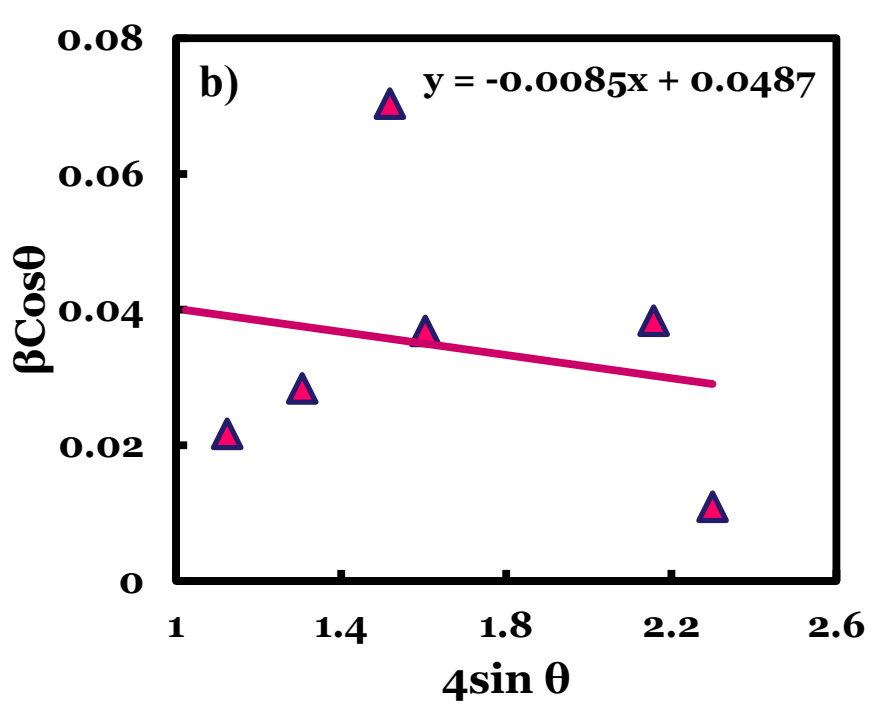

Slope $=-\mathbf{0 . 0 0 8 5}$ Particle size $=\mathbf{2 . 8 4 9 3} \mathbf{n m}$ 


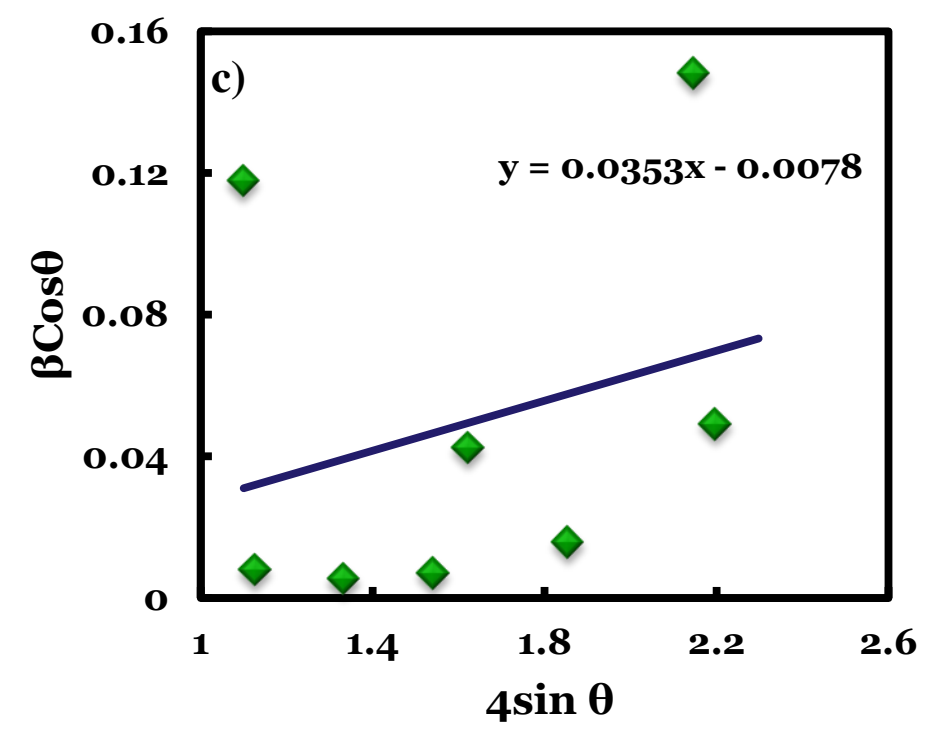

Slope $=0.0353$ Particle size $=0.0078 \mathrm{~nm}$

Figure 3. (a-c) Williamson-Hall plots of CuZn alloy

Table.1. Textural parameters of the $\gamma-\mathrm{CuZn}$ alloy from XRD, TEM and UV- DRS studies

\begin{tabular}{|c|c|c|c|c|c|c|c|}
\hline \multirow[b]{2}{*}{ Sample } & \multirow[b]{2}{*}{ Composition } & \multirow[b]{2}{*}{$\mathrm{pH}$} & \multirow{2}{*}{$\begin{array}{l}\text { Scherrer } \\
\text { Method } \\
\text { D (nm) }\end{array}$} & \multicolumn{2}{|c|}{$\begin{array}{l}\text { Williamson-Hall } \\
\text { Method }\end{array}$} & \multirow{2}{*}{$\begin{array}{l}\text { TEM } \\
\text { Grain size } \\
\text { D (nm) }\end{array}$} & \multirow{2}{*}{$\begin{array}{l}\mathrm{E}_{\mathrm{g}} \\
(\mathrm{eV})^{-}\end{array}$} \\
\hline & & & & $\begin{array}{l}\text { Particle } \\
\text { size } \\
\text { D (nm) }\end{array}$ & Strain $\varepsilon$ & & \\
\hline I & $1: 1$ & 3.5 & 16 & 11 & -0.0009 & 10 & 3.38 \\
\hline II & $1: 2$ & 3.6 & 5.6 & 2.8 & -0.0085 & 5 & 2.73 \\
\hline III & $2: 1$ & 3.8 & 10 & 17.8 & +0.0353 & 12 & 3.46 \\
\hline
\end{tabular}

\subsection{Preferred Orientation (Texture Coefficient)}

The texture (preferred orientation) of the $\gamma-\mathrm{CuZn}$ was quantitatively analysed using Harris method of determining both the degree of preferred orientation and texture coefficient as shown in the fig. 4. 


$$
T C=\frac{I(h k l) / I_{0}(h k l)}{\frac{1}{N} \sum I(h k l) / I_{0}(h k l)} \times 100 \%
$$

Where $\mathrm{I}(\mathrm{hkl})_{i}$ is the measured peak intensity of the $(\mathrm{hkl})$ plane for the $\mathrm{i}^{\text {th }}$ peak, $\mathrm{I}_{0}(\mathrm{hkl})_{i}$ the reference (JCPDS) intensity for the corresponding(hkl), and N reference the total diffraction peaks in the diffractogram. For arbitrarily oriented crystallites, the texture coefficient is always unity $\left(T C_{(h k l)}=1\right)$ however deviation from unity point toward the preferred growth of the nano alloy. The TC calculations revealed the (222) plane as the preferred angle for its random orientation in the $\gamma-\mathrm{CuZn}$ bimetallic alloy and the results are presented in table 2 .

Table. 2. The texture coefficient for (101) plane for the Zn-nano hexagons synthesized at different reaction parameters

\begin{tabular}{|l|l|ll|l|}
\hline Sample & FWHM (222) & $\begin{array}{l}\text { Grain } \\
(\mathrm{nm})\end{array}$ & Size & $\mathrm{TC}_{222}$ \\
\hline I & 0.3493 & 11 & 1.42 \\
\hline II & 1.2961 & 2.8 & 0.55 \\
\hline III & 7.0261 & 17.8 & 0.68 \\
\hline
\end{tabular}




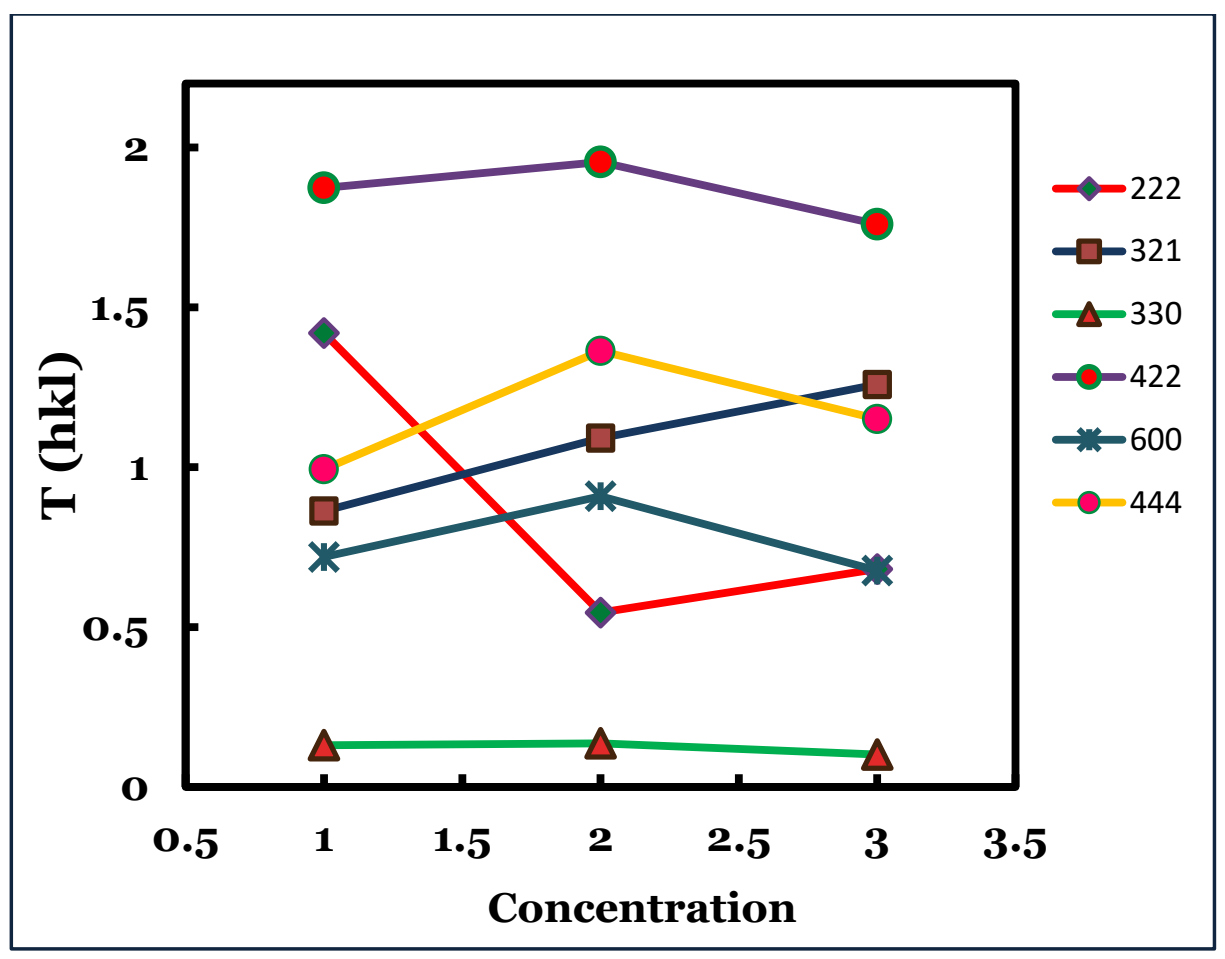

Figure 4. Texture coefficient $\mathrm{T}(\mathrm{hkl})_{i}$ of the $\mathrm{CuZn}$ bimetallic alloy obtained at different electrolytic concentration

\subsection{Textural morphology of the bimetallic nano alloy}

Textural morphology of the bimetallic nano alloy was further investigated by high resolution transmission electron microscopy. TEM micrographs served as an important tool that exposes the structural defects and interfaces between the bimetallic nano catalysts through lattice fringes. The HR-TEM micrographs of CuZn bimetallic with average particle size of $5 \mathrm{~nm}$ are shown in Fig. 5. The images obtained for the bimetallic nano alloy at optimum conditions revealed that the particles are in cubic structure and are clustered together. These findings also indicated that the bimetallic alloy is in the crystalline form which is revealed from the lattice fringes. The atomic arrangements in the lattice fringes show the defect in the lattice structure of pure copper or zinc that confirms the CuZn alloy formation. As can be seen in the TEM micrographs the particles 
are dispersed randomly due to the synthesis conditions and more or less agglomerations also existed. However, moderately agglomerated particles, in addition dispersed particles, were seen in the micrographs. HR-TEM images of CuZn bimetallic nanocrystal showing the $3.82 \AA$ lattice spacing very clearly. In contrast, sharpness and position of the atoms are clearly explained from the TEM images.
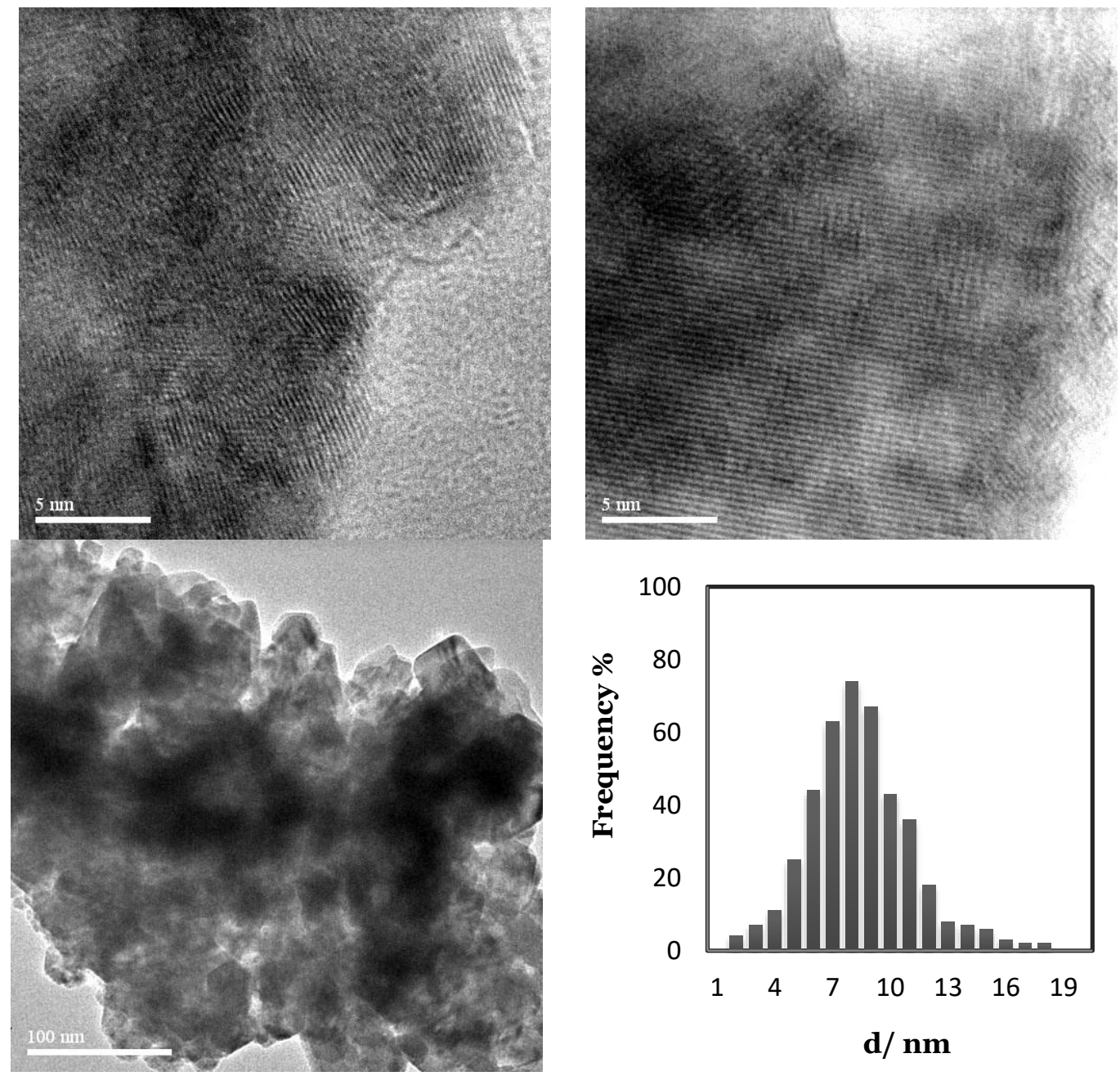

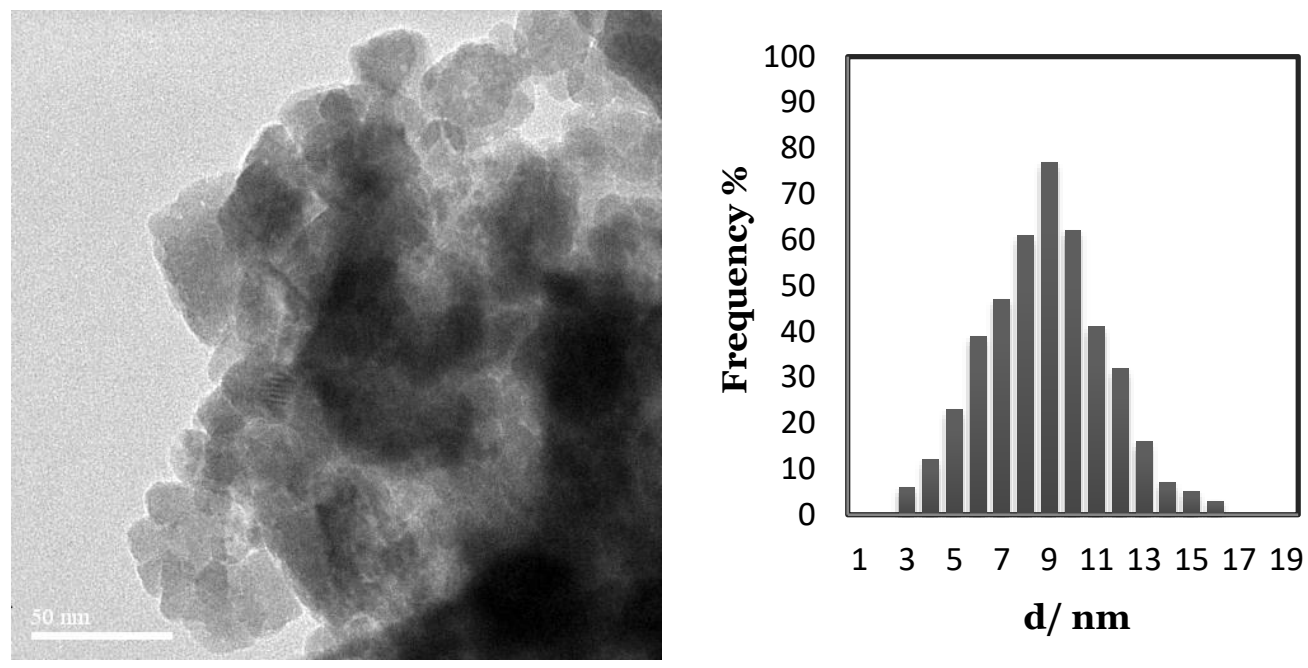

Figure 5. TEM micrographs and particle size histogram of CuZn nano alloy prepared from eletrodeposition method

\subsection{X-ray photoelectron spectroscopy analysis of nano alloy}

$\mathrm{X}$-ray photoelectron spectroscopy was performed to get the chemical confirmation and nature of oxidation state of nano alloy. XPS analysis makes available information about the composition of the $\mathrm{CuZn}$ by quantifying the nano alloy ratio. The line position and nature of $\mathrm{Cu}$ L3 VV, Zn L3 M4, 5 M4, 5 reveal that the nano composition is metallic for the $\gamma$-CuZn nano alloy obtained from the electrodeposition. The survey spectrum revealed the presence of the entire element in the electrodeposition $\gamma-\mathrm{Cu}_{5} \mathrm{Zn}_{8}$ bimetallic nano alloy. The $2 \mathrm{p}$ line strengths disclose an average configuration of nano alloy of $\mathrm{Cu}$ : $\mathrm{Zn}$. The satellite feature in the $\mathrm{Cu} 2 p_{3 / 2}$ line discloses that $\mathrm{Cu}$ is in the reduced form. Fig. 6 shows the normalized and background corrected images of the alloy and the Auger spectrum meant for $\mathrm{Cu} / \mathrm{Zn}$ is shown in fig. $6(\mathrm{~b}-\mathrm{c})$. This information evidently indicates that $\mathrm{Cu}: \mathrm{Zn}$ nano brass is present in the reduced form in the optimized reaction conditions. 
However, more pronounced $\mathrm{Zn} / \mathrm{Cu}$ signals are observed which can be attributed to the reduced form of these metals in the alloy or lower amount of oxidized form which in turn intensify the sensitivity to bimetallic $\gamma$-CuZn recognition. This stresses the advantages of adopting this method for the nano alloy synthesis. Furthermore, it is perceived that $\mathrm{Zn} \mathrm{L}_{3} \mathrm{M}_{4}$, the $5 \mathrm{M} 4,5$ stroke showed a shift towards the lower binding energies as a consequence the reduction of metal ions is carried out at room temperature.

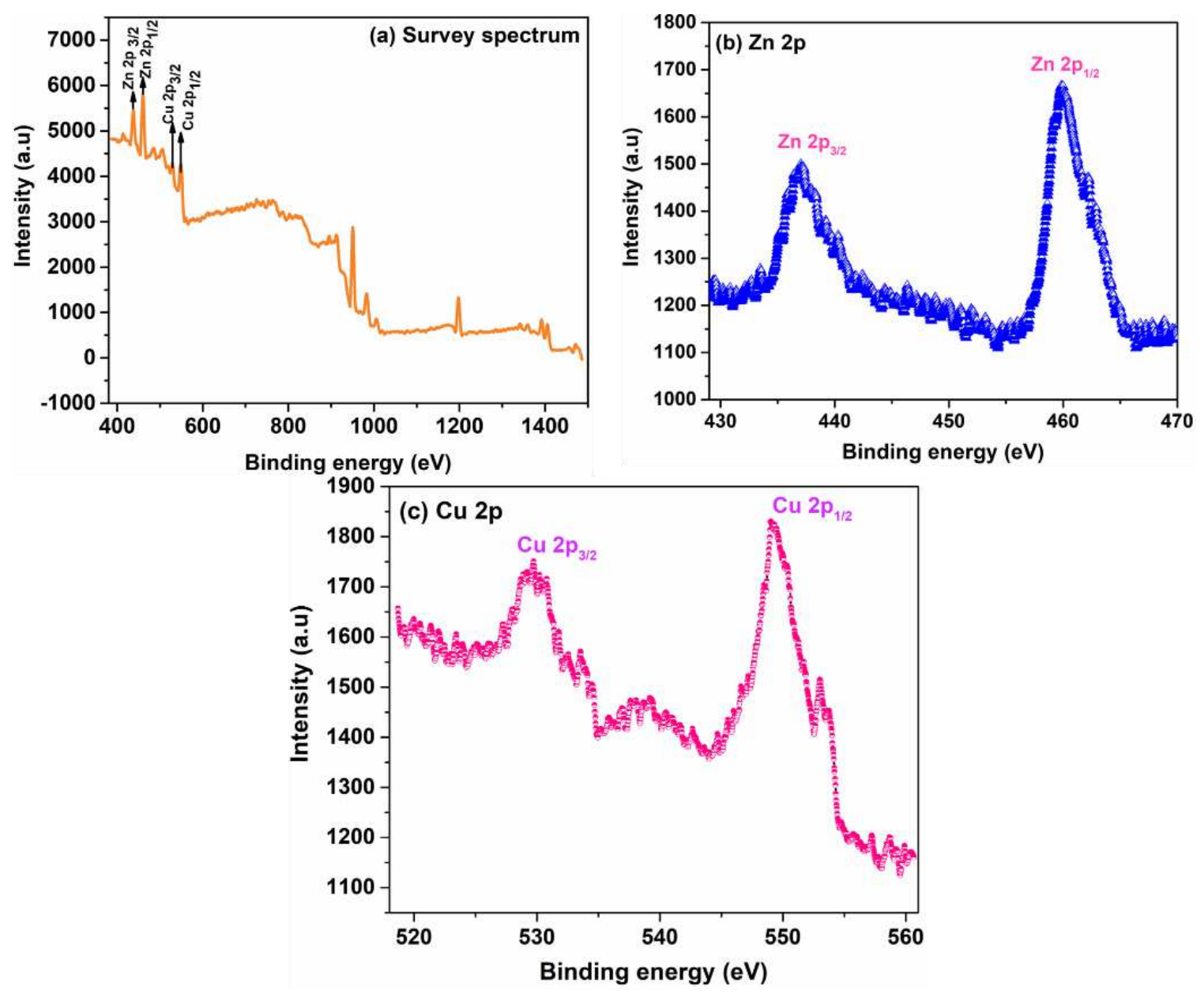

Figure 6. XPS analysis of $\mathrm{CuZn}$ alloy (a) survey spectrum of $\gamma$-Cu5Zn8 bimetallic nano alloy (b) high resolution XPS 2 p spectrum showing $\mathrm{Zn} 2 \mathrm{p}_{3 / 2}$ peak at 443 and $2 \mathrm{p}_{1 / 2}$ peak at $465 \mathrm{eV}$, (c) spectrum for $\mathrm{Cu} 2 \mathrm{p}_{3 / 2}$ peak at 535 and $2 \mathrm{p}_{1 / 2}$ peak at $555 \mathrm{eV}$ 


\subsection{FT-IR spectral analysis}

The FTIR study has been carried out to identify the elements and their phase, which is expected to be adsorbed on $\gamma$-CuZn nano surface during sample preparation. The infrared spectrum of the CuZn bimetallic nanoparticle synthesized from electrodeposition technique is shown in Fig. 7. The IR spectrum showed a broad and low intense peak near mid-IR region. In the case of metal, the adsorbed impurities on the surface of the particles are normally found near the mid-IR region. This can be ascribed to the $\mathrm{CO}_{2}$ concentration from the atmosphere on the surface of the metal and it can be understood from the spectrum that, the high surface area of the nano alloy facilitated the ease of adsorption of more $\mathrm{CO}_{2}$ on the surface and the major frequency of absorption is seen near $2036 \mathrm{~cm}^{-1}$ [27]. A small absorption peak near $1400 \mathrm{~cm}^{-1}$ can be assigned to the shift in peak position. The restoring force created by the surface polarization charge was responsible for the change in the frequency. The samples show a common band $3450 \mathrm{~cm}^{-1}$. This band accounts for the hydroxyl stretching frequency $[v(\mathrm{OH})]$, due to surface adsorbed water molecule. The low intense peak at $1620 \mathrm{~cm}^{-1}$ indicates the trace amounts of the water molecule that is being adsorbed on the surface of during sample preparation with $\mathrm{KBr}$ pellet. The strong, intense peaks around $2000 \mathrm{~cm}^{-}$

${ }^{1}$ can be attributed to the presence of pollutants that are strongly adsorbed on the surface of the bimetallic alloy. No other prominent peaks corresponding to copper or zinc oxide were found which data is in good agreement with the XPS. However, considering the FTIR spectrum, it is most likely due to the formation of alloy instead of pure metals. 


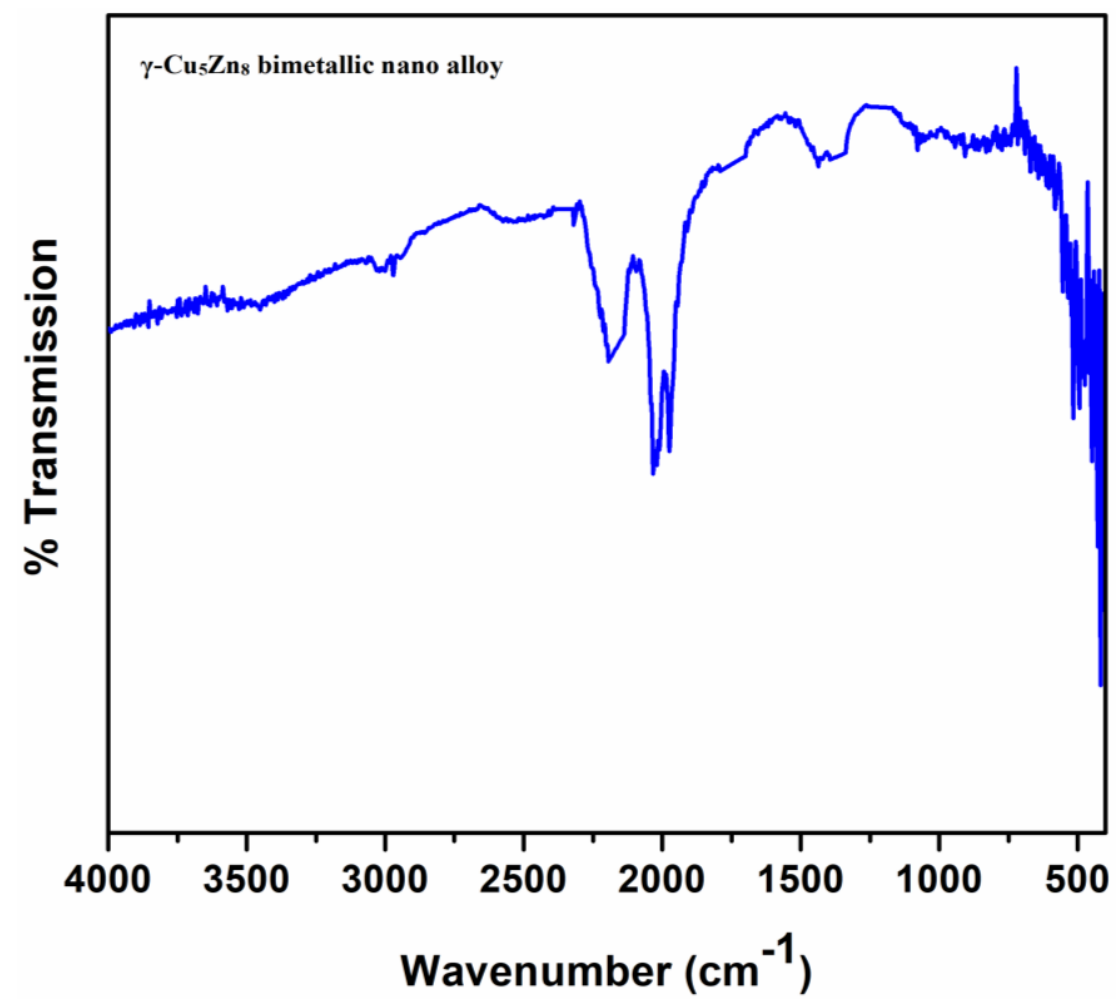

Figure 7. FT-IR spectrum of CuZn alloy

\subsection{EDAX spectrum of $\gamma$-CuZn bimetallic alloy}

The EDAX spectrum mapping is presented in Fig. 8 clearly established the uniform distribution of copper and zinc in the $\gamma$-CuZn bimetallic alloy. This result is in good agreement with TEM observations. It was also observed that the atomic percentage of both the metals in the nano alloy synthesized from varied reaction parameters showed appreciable change. Surprisingly the atomic composition of $\mathrm{Cu}$ and $\mathrm{Zn}$ in the nano alloy did not show much difference as the applied current and $\mathrm{pH}$ of the electrolyte are varied. However, the EDX analysis displayed CuZn ratio of 28:35 with little oxide formation indicating that the electrodeposition is highly influenced by the concentration of metal ions in the electrolyte at low current densities as shown in the Fig. 8. These findings indicate that $\mathrm{Cu}$-ions being nobler than $\mathrm{Zn}$ have gotten reduced at the cathode first since the migration of $\mathrm{Cu}^{2+}$ ions faster at higher current densities and higher molar concentrations. The 
EDX results show that the alloy contains on average about 60 mole $\%$ of copper and 40 mole $\%$ $\mathrm{Zn}$. It is also concluded that the large amount of $\mathrm{Cu}$ in the alloy apparently makes the $\mathrm{CuZn}$ nano alloy highly stable.

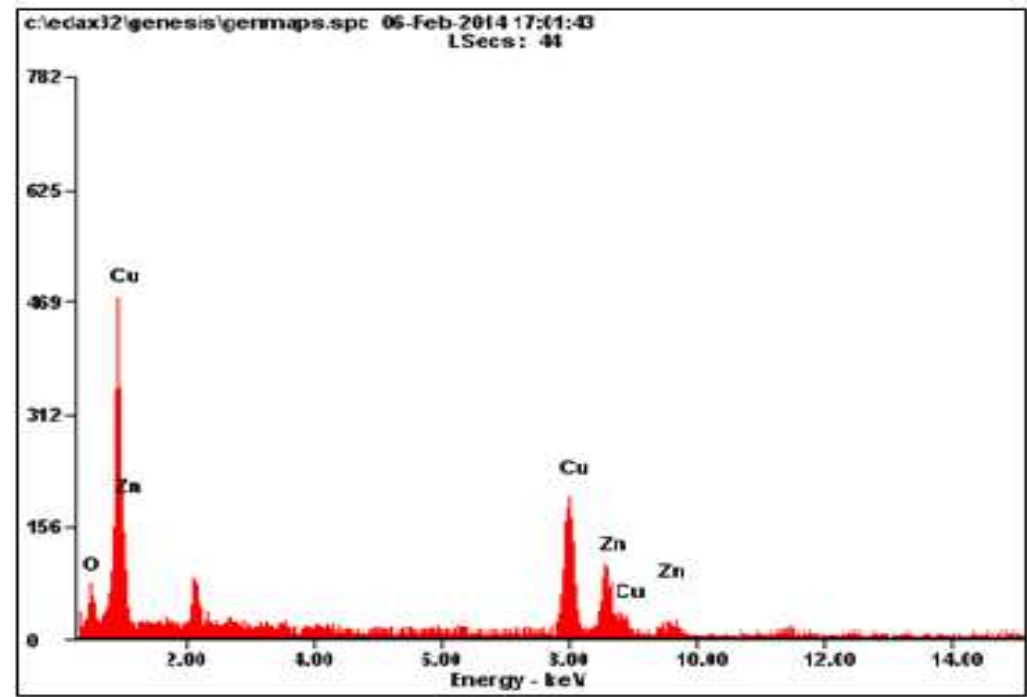

\begin{tabular}{||l|l|l||}
\hline \multicolumn{1}{|c|}{ Element } & Wt\% & At\% \\
\hline OK & 5.42 & 18.25 \\
\hline CuK & 53.46 & 48.51 \\
\hline ZnK & 35.12 & 33.24 \\
\hline Matrix & Correction & ZAF \\
\hline \hline
\end{tabular}

Figure 8. EDX analysis of CuZn alloy

\subsection{Diffuse reflectance spectroscopy analysis}

Diffuse reflectance spectroscopy (DRS) was employed to disclose the optical properties and electrical band gap of $\gamma$-CuZn nano brass. Optical absorption is the characteristic property of the metals in particular for the bimetallic nano alloy systems. According to Mie theory, isotropic nanomaterials alone show single absorption band in the UV-Visible spectrum, while dispersed 
anisotropic particles with different surface morphologies exhibit two or even three absorption bands when the particle size falls below $10 \mathrm{~nm}$. Fig. 9 a) demonstrates the absorption spectrum converted from the diffuse reflectance measurements for CuZn bimetallic alloy obtained from optimum reaction conditions. A quadrupole resonance band can be seen at shorter wavelength indicating the small particle size of the CuZn bimetallic nano alloy. The sharp peak with high intensity confirmed the formation of CuZn alloy and the Shift in peak position is observed for the CuZn bimetallic towards the shorter wavelength. On the basis of the observed surface plasmon band, it is observed that structure of the formed $\mathrm{CuZn}$ is cubic. The copper predominant alloy absorbed around $458 \mathrm{~nm}$, while the alloy with Zn-rich composition is observed in the low wavelength.

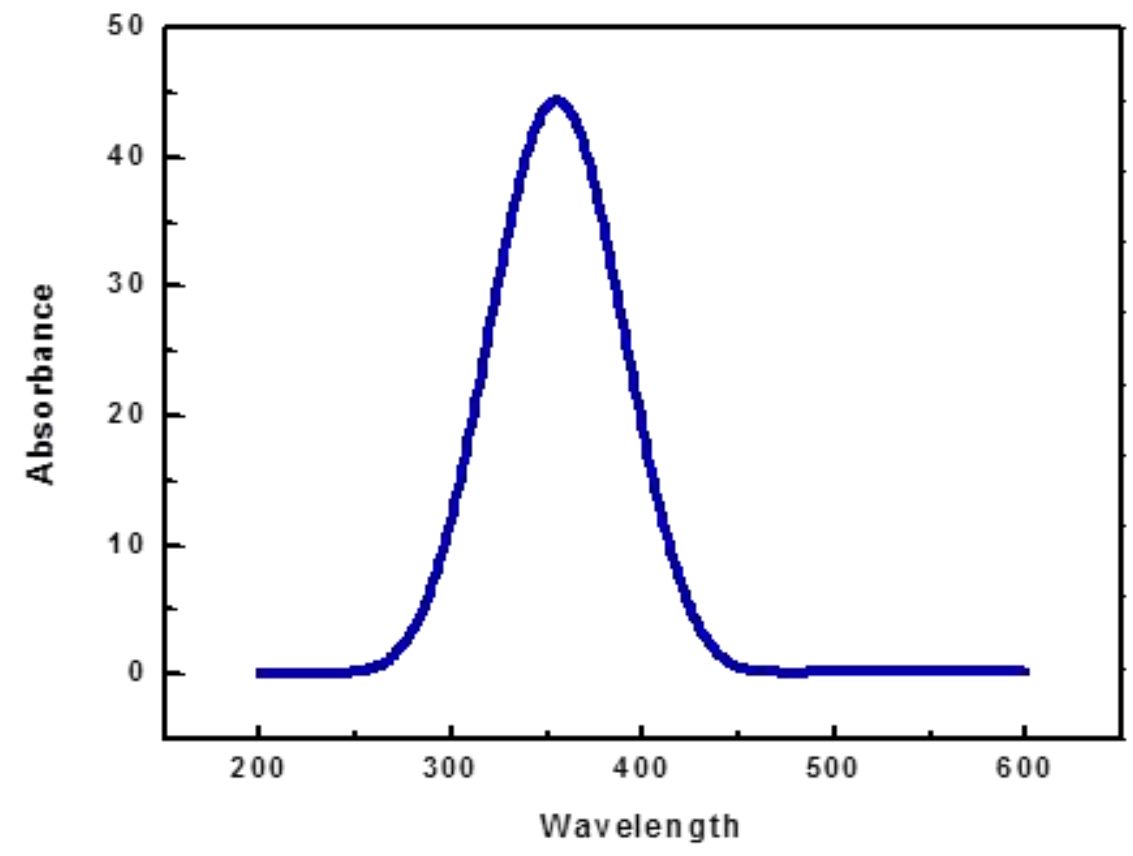

Figure 9. ( a) UV-DRS spectrum of CuZn nano alloy

These techniques, possibly will not define the accurate determination of the band gap though the peak position and it can be concluded that the energy gap is related to the electrical conductance of the materials. However, it is possible to extract the band gap unambiguously from 
the reflectance spectra by means of the Kubelka-Munk algorithm $F(R)=\frac{(1-R)^{2}}{2 R}=\frac{k}{s}$. Where $\mathrm{R}$ is absolute reflectance, $\mathrm{k}$ is molar absorption co-efficient and $\mathrm{S}$ is scattering co-efficient. The curve between the quantity $F(R)$ hv0.5 on the abscissa and photon energy (hv) on the ordinate is drawn. The energy gap $\left(E_{g}\right)$ obtained from the intercept of a tangent drawn to the point of inflection on the curve on the horizontal axis. Figure $9 \mathrm{~b}$ ) provides the band gap energy $\left(E_{g}\right)$ value of $2.73 \mathrm{eV}$ for the electrodeposited CuZn alloy. The narrow band gap illustrate that the nano alloy is capable of absorbing visible light.

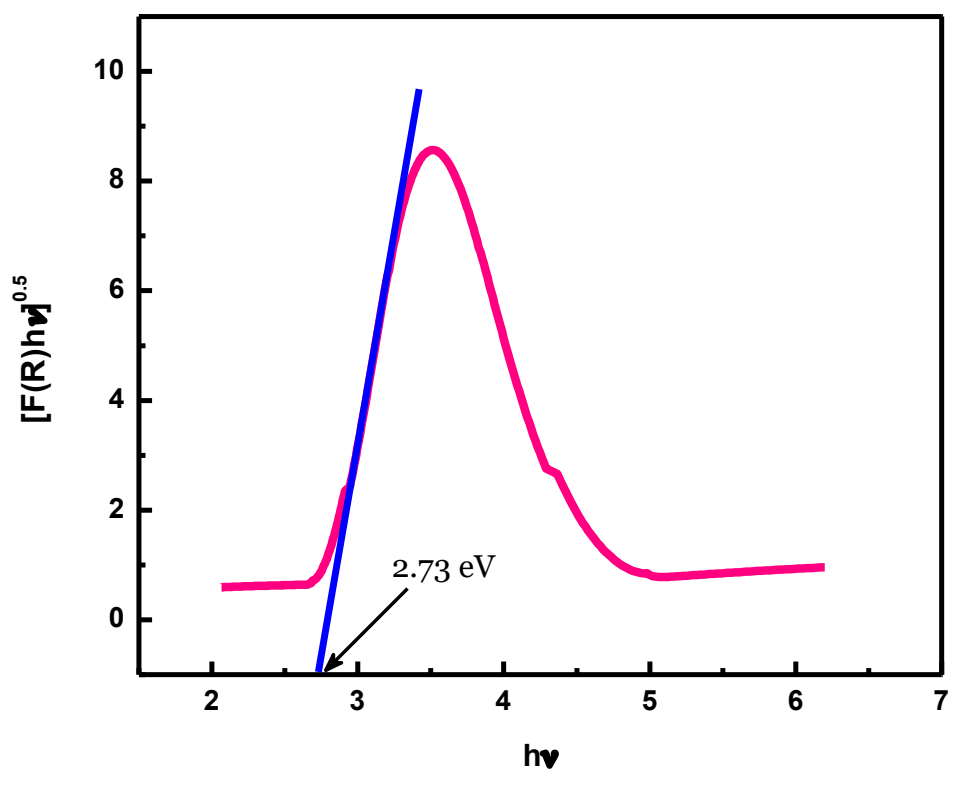

Figure 9. (b) Kubelka-Munk plot of CuZn nano alloy showing band gap energy 


\subsection{Antibacterial Efficacy}

Antimicrobial activity of CuZn bimetallic synthesized by the electrodeposition process was studied against two gram-positive microorganisms S. Aureus B. Subtilis and gram-negative pathogens K. Pneumonia and E. coli which are dominant causative agents for life-threatening diseases. The antibacterial efficacy of CuZn bimetallic alloy was evaluated via the well diffusion assay. The agar plates were inoculated by an inoculum of the test microorganisms then the $\mathrm{CuZn}$ bimetallic of different concentrations were placed on the agar surface followed by incubation under laboratory conditions and the results are shown in fig. 10.

CuZn bimetallic showed a slightly higher efficacy against the Staphylococcus aureus compared to other tested bacteria. Interestingly the amount required for growth inhibition (MIC) for gram-positive, gram-negative bacteria together was almost equal, suggesting that the antimicrobial efficacy of CuZn against the bacteria is metabolic pathways of bacteria.

Of all the mechanisms proposed, enzymatic inhibited, Porin mutations, efflux pumps, penicillin-binding protein modifications, and target changes are showing a high frequency of clinical isolate. The most common mechanism is enzymatic inhibition. The CuZn bimetallic exhibited excellent antibacterial efficacy against the tested microorganisms. The mechanism of toxicity towards the microorganisms for CuZn alloy is different from those of copper and zinc ions and showed a high level of membrane-damaging nature of the microorganisms. 

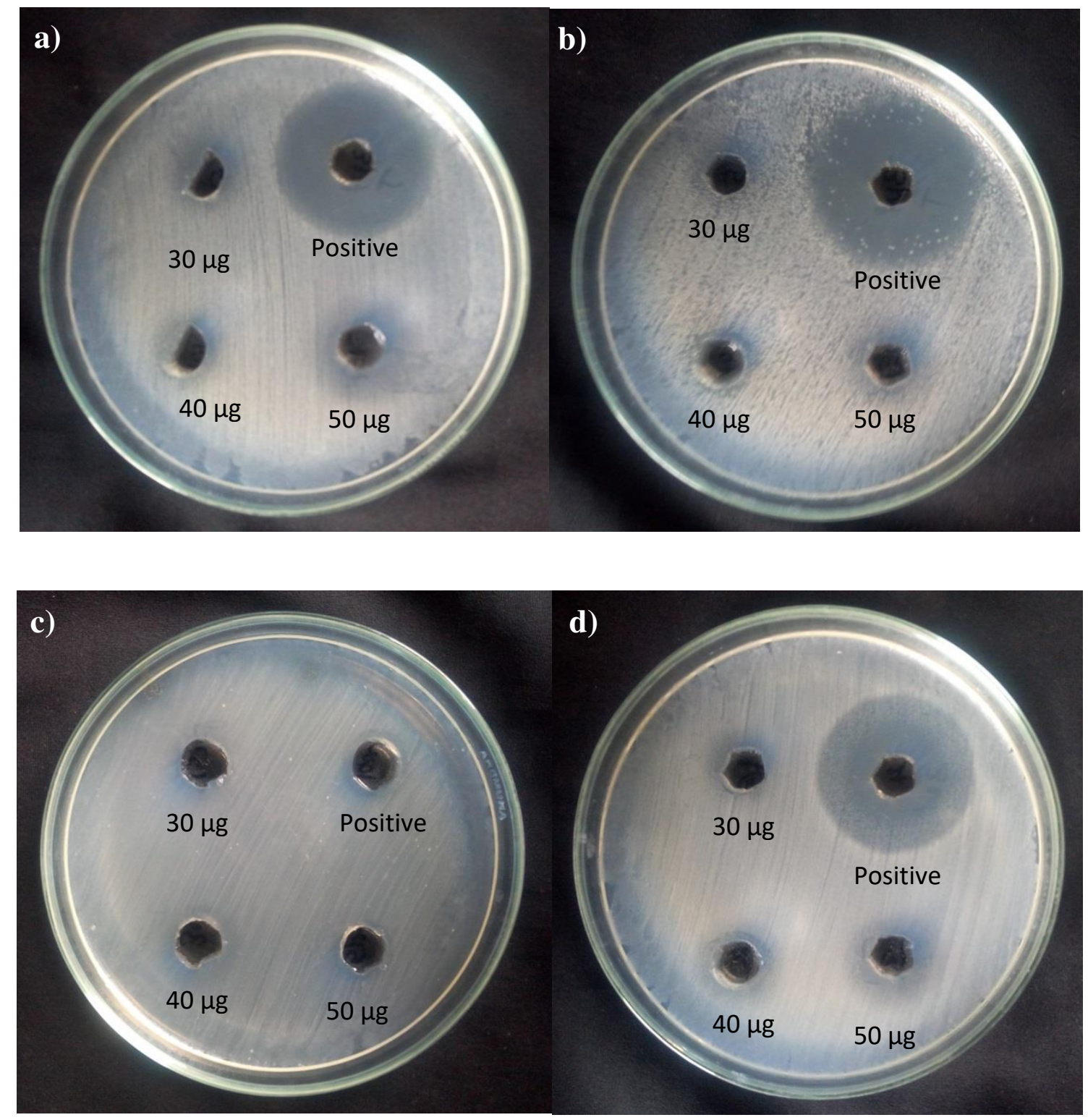

Figure 10. Well diffusion assay showing Zone of inhibition of $\mathrm{CuZn}$ alloy at optimum reaction conditions against a) Klebsiella pneumoniae b) Escherichia Coli, c) Bacillus Subtilis and d) Staphylococcus Aureus

\subsection{Photocatalytic process of $\gamma$-CuZn Bimetallic alloy}

To delineate the absorption of visible light activity of $\gamma$-CuZn photocatalyst we selected methyl orange (MO) dye as the model contaminant with the absorption peak at $455 \mathrm{~nm}$. The UV 
- Visible absorption spectra for the photocatalytic degradation of MO dye is shown in Figure 11 a). The photo-degradation process was optimized with $0.01 \mathrm{~g} / \mathrm{L}$ nano-catalyst and $50 \mathrm{mg} / \mathrm{L}$ of dye concentration. Complete degradation of MO was accomplished in $60 \mathrm{~min}$. as given in the figure 11 a) the absorption peak intensity of MO dye at $460 \mathrm{~nm}$ was decreased gradually within $60 \mathrm{~min}$ indicating the degradation of MO into harmless fragments. The photocatalytic degradation involves the adsorption of UV radiation by the heterogeneous nano catalyst followed by the degradation through holes (positive charge) from the nanocatalyst caused by solar light irradiation. The positive holes oxidise the methyl orange molecule by direct electron transfer to the hydroxyl radical generated from the water molecule aimed at the active photocatalysis. Fig. 11 (b) shows the kinetic performance of $\gamma-\mathrm{CuZn}$ photocatalyst. The linear fit of $\operatorname{lnC} \mathrm{C}_{0} / \mathrm{C}$ vs irradiation time confirms first order kinetics (pseudo) and the slope drawn gives the first order rate constant value $0.02689 / \mathrm{min}$.

\subsection{Effect of catalyst dose}

Fig.11.b shows the effect of catalytic load on the photocatalytic degradation reaction. In order to evaluate the optimal dosage amount of the prepared catalysts; the catalytic weight was varied and the optimized amount was found to be $10 \mathrm{mg} / \mathrm{L}$. Moreover, below this optimum load, leads to increasing surface active site which results rapid degradation. Above the optimum load, increases the scullery turbidity, as well the light penetration decreases and thus, availability of $\mathrm{OH}$ - and $\mathrm{O}_{2}{ }^{-}$- becomes minimal.

\subsection{Effect of dye concentration}

Figure (11 c) shows the effect of dye concentration on the photocatalytic activity of CuZn bimetallic alloy NPs. As the concentration of dye increases, with increasing time taken for 
complete degradation increases. The results reveals that, at increased concentrations more dye molecules are adsorbed on to the surface of catalyst resulting in decreased active sites on the catalyst (lesser number of hydroxyl and superoxide radicals) and thereby, reduced light penetration.

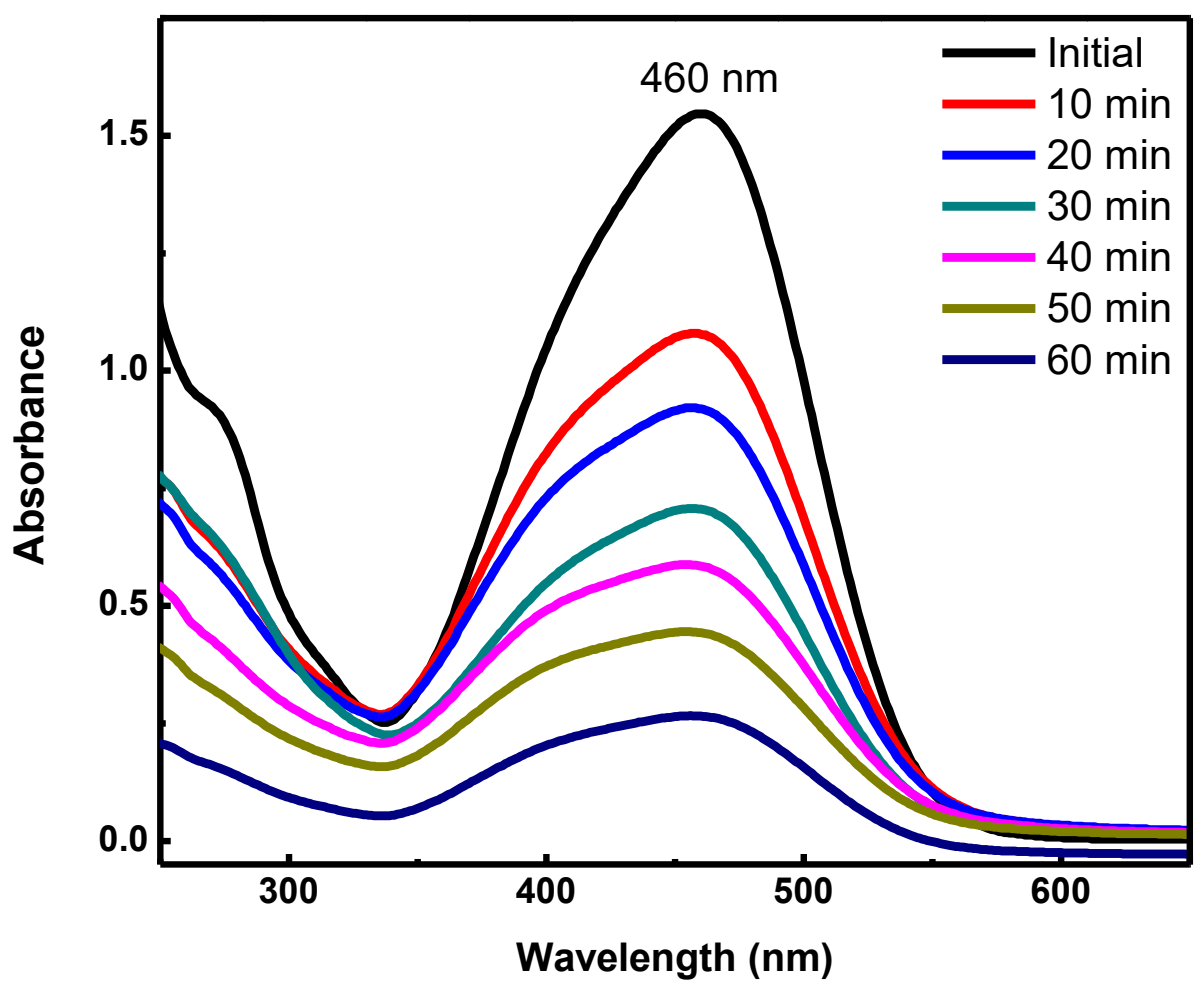

Figure 11. (a) UV-visible absorption spectra showing the degradation of Methyl Orange upon sunlight irradiation using $\gamma$-CuZn alloy 


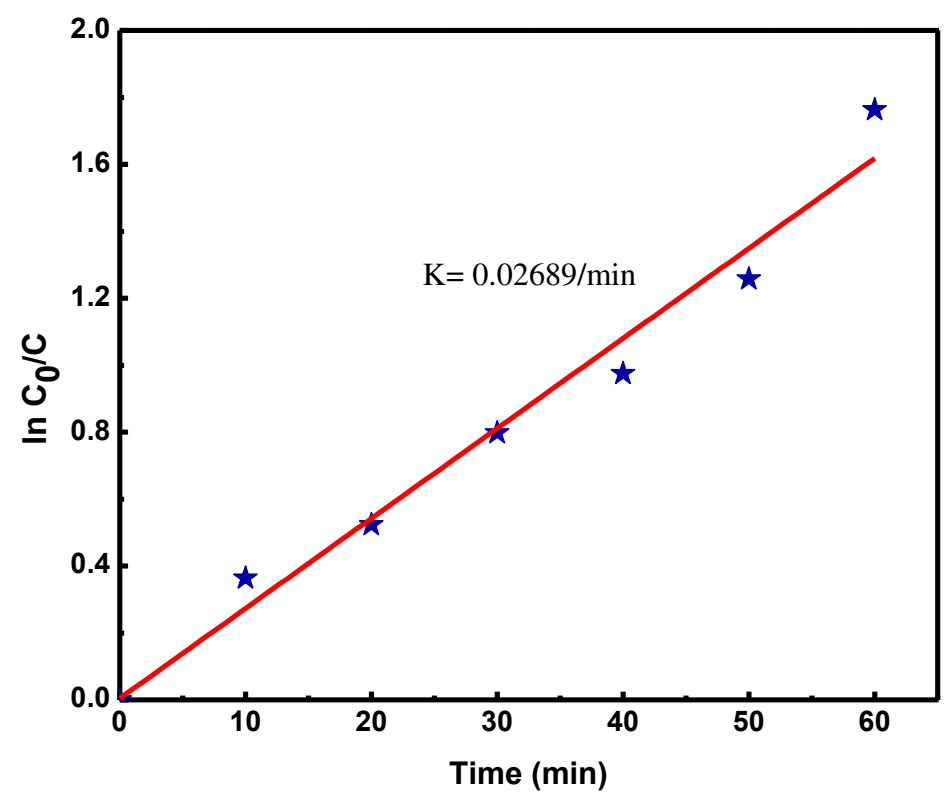

Figure 11. (b) Pseudo first order kinetics for photocatalytic degradation of methyl orange dye using $\gamma-\mathrm{CuZn}$ nanocatalyst

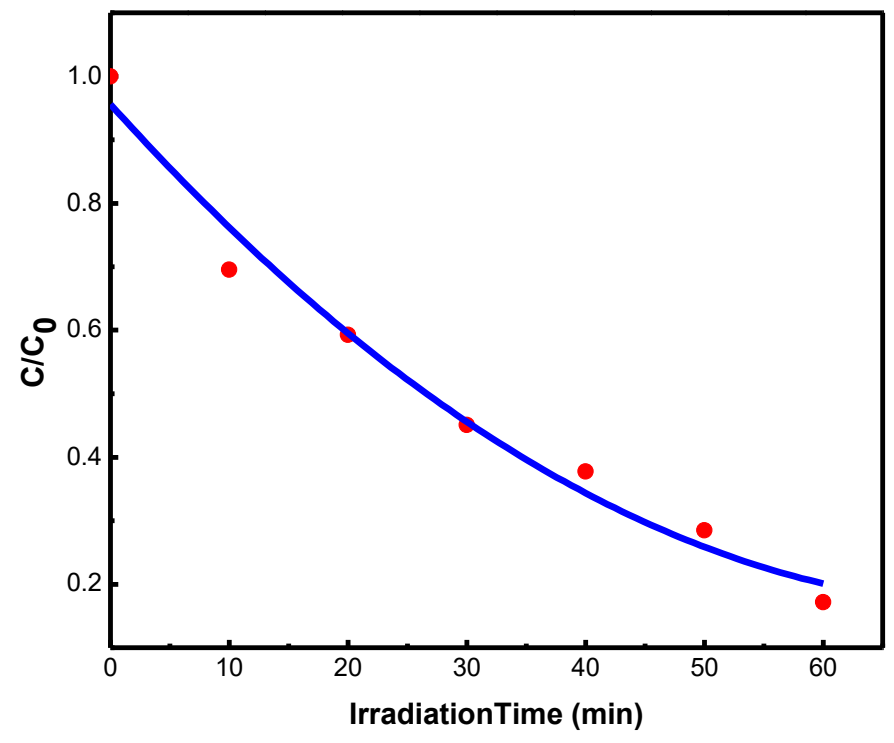


Figure 11. (c) photocatalytic degradation profile of MO by CuZn nano alloy

\section{CONCLUSION}

These results illustrate the fast, simple and scalable technique meant for the synthesis of bimetallic nano alloy from direct electro-deposition. The electron microscope results revealed the cubic surface morphology and the XPS result authenticated the presence of elemental copper and zinc in nano-brass. Powder XRD revealed the $\gamma$-phase of the electrodeposited nano brass. The optical response for the nano alloy has been proved by diffuse reflectance spectroscopy and the photocatalytic efficiency of the nano brass can be attributed to the surface plasmon response. An amazing photocatalytic activity was accomplished for the bimetallic nano alloy under the visible light irradiation that highlights the versatility of nano catalyst fabricated by the electrochemical method. The antibacterial efficacy of the as-synthesized nano alloy showed the enhanced synergistic effect against the micro-organisms. Our studies showed the monodispersed nano alloy can be a potential candidate for the environmental issues in the current prevalence. In conclusion, the results shed light on a facile and cost-effective method for alloy NPs for superior structural properties to the copper and zinc NPs with wide range of applications.

\section{Acknowledgments}

The Basic Science Research Program supported this study through the National Research Foundation of Korea (NRF) funded by the Ministry of Education (2020R1I1A3054816).

Competing Interests: The authors declare no competing interests. 


\section{References}

1. R. Ferrando, J. Jellinek, R. L. Johnston, Nano alloys: from theory to applications of alloy clusters and nanoparticles, Chem. Rev. 108 (2008) 845-910.

2. T. Omori, K. Ando, M. Okano, X. Xu, Y. Tanaka, I. Ohnuma, R. Kainuma, K. Ishida, Superelastic Effect in Polycrystalline Ferrous Alloys, Science. 333 (2011) 68-71.

3. J. A. Rodriguez, D. W. Goodman, The nature of the metal-metal bond in bimetallic surfaces, Science. 257 (1992) 897-903.

4. J. Tanori, N. Duxin, C. Petit, I. Lisiecki, P. Veillet, M. P. Pileni, Synthesis of nanosize metallic and alloyed particles in ordered phases, Colloid Polym. Sci. 273 (1995) 886-892.

5. M. Sankar, Q. He, S. Dawson, E. Nowicka, L. Lu, P. C. A. Bruijnincx, A. M. Beale, C. J. Kiely, B. M. Weckhuysen, Supported bimetallic nano-alloys as highly active catalysts for the one-pot tandem synthesis of imines and secondary amines from nitrobenzene and alcohols, Catal. Sci. Technol. 6 (2016) 5473-5482.

6. R. C. Tiruvalam, J. C. Pritchard, N. Dimitratos, J. A. Lopez-Sanchez, J. K. Edwards, A. F. Carley, G. J. Hutchings, C. J. Kiely, Aberration corrected analytical electron microscopy studies of sol-immobilized $\mathrm{Au}+\mathrm{Pd}, \mathrm{Au}\{\mathrm{Pd}\}$ and $\operatorname{Pd}\{\mathrm{Au}\}$ catalysts used for benzyl alcohol oxidation and hydrogen peroxide production, Faraday Discuss. 152 (2011) 63-86.

7. F. Tao, M. E. Gras, Y. Zhang, D. R. Butcher, J. R. Renzas, Z. Liu, J.Y. Chung, B. S. Mun, M. Salmeron, G.A. Somorjai, Reaction-driven restructuring of Rh-Pd and Pt-Pd core-shell nanoparticles, Science. 322 (2008) 932-934.

8. B. Lim, M. Jiang, P. H. Camargo, E. C. Cho, J. Tao, X. Lu, Y. Zhu, Y. Xia, Pd-Pt bimetallic nanodendrites with high activity for oxygen reduction, Science. 324 (2009) 1302-1305. 
9. M. A. Zagrebin, V. V. Sokolovskiy, V. D. Buchelnikov1, M. A. Klyuchnikova, First Principles Investigation of Magnetic Properties of Fe-Ni-Mn-Al Heusler Alloys, Physics Procedia. 75 (2015) 1427-1434.

10. E. Gonzalez, J. Arbiol, V. F. Puntes, Carving at the nanoscale: sequential galvanic exchange and Kirkendall growth at room temperature, Science. 334 (2011) 1377- 1380.

11. J. Yang, S. Deng, J. Lei, H. Ju, S.Gunasekaran, Electrochemical synthesis of reduced graphene sheet-AuPd alloy nanoparticle composites for enzymatic biosensing, Biosens. Bioelectron. 29 (2011) 159-166.

12. J. Yang, W. -D. Zhang, S. Gunasekaran, An amperometric non-enzymatic glucose sensor by electrodepositing copper nanocubes onto vertically well-aligned multi-walled carbon nanotube arrays, Biosens. Bioelectron. 26 (2010) 279-284.

13. J. C. Colmenares, R. Luque, Heterogeneous photocatalytic nanomaterials: prospects and challenges in selective transformations of biomass-derived compounds, Chemical Society Reviews. 43 (2014) 765-778.

14. M. Sahni, S. Mukhopadhyay, , R. M. Mehra, S. Chauhan, , P. C. Sati, M. Kumar, M. Singh, and N. Kumar, Effect of $\mathrm{Yb} / \mathrm{Co}$ co-dopants on surface chemical bonding states of BiFeO3 nanoparticles with promising photocatalytic performance in dye degradation, J. Phys. Chem. Solids. (2021) 109926.

15. X. Li, J. Yu, M. Jaroniec, Hierarchical photocatalysts, Chemical Society Reviews. 45 (2016) 2603-2636.

16. N. Zhang, S. Q. Liu, Y. J. Xu, Recent progress on metal core@ semiconductor shell nanocomposites as a promising type of photocatalyst, Nanoscale. 4 (2012) 2227-2238. 
17. X. Zhang, Y. Wang, B. Liu, Y. Sang, H. Liu, Heterostructures construction on $\mathrm{TiO}_{2}$ nanobelts: A powerful tool for building high-performance photocatalysts, Applied Catalysis B: Environmental. 202 (2017) 620-641.

18. Y. F. Lin, Y. J. Hsu, Interfacial charge carrier dynamics of type-II semiconductor nanoheterostructures, Applied Catalysis B: Environmental. 130 (2013) 93-98.

19. N. Toshima, T. Yonezawa, Bimetallic Nanoparticles-Novel Materials for Chemical and Physical Applications, New J Chem. 22 (1998) 1179-1201.

20. C. Jo, J. Lee, Y, Jang, Electronic and Magnetic Properties of Ultrathin FeCo Alloy Nanowires, Chem Mater. 17 (2005) 2667-2671.

21. A. K. Jaiswal, S. Singh, A. Singh, R.R. Yadav, P. Tandon, Fabrication Of Cu/Pd Bimetallic Nanostructures With High Gas Sorption Ability Towards Development Of LPG Sensor, Mat. Chem. Phys. 154 (2015) 16-21.

22. S. Chen, H. Zhang, L. Wu, Y. Zhao, C. Huang, Controllable Synthesis of Supported CuM (M = Pt, Pd, Ru, Rh) Bimetal Nanocatalysts And Their Catalytic Performances, J. Mater Chem. 2012, 22, 9117-9122.

23. K. Karthik, , S. Dhanuskodi, , C. Gobinath, , S. Prabukumar and S. Sivaramakrishnan, Nanostructured CdO-NiO composite for multifunctional applications, J. Phys. Chem. Solids. 112, (2018) 106-118.

24. Z. H. Shih, C. W. Wang, G. Xu, H. T. Chang, Porous Palladium Copper Nanoparticles for the Electrocatalytic Oxidation of Methanol in Direct Methanol Fuel Cells, J Mater Chem A. 1 (2013) 4773-4778. 
25. L. Zhang, F. Hou, Y. Tan, Shape-tailoring of CuPd nanocrystals for enhancement of electro-catalytic activity in oxygen reduction reaction, Chem Commun (Camb). 48 (2012) $7152-7154$.

26. S. H. Y. Lo, T. Y. Chen, Y. Y. Wang, C. C. Wan, J. F. Lee, A Mechanism Study On The Synthesis Of Cu/Pd Nanoparticles With Citric Complexing Agent, J Phys Chem. C. 111 (2007) 12873-12876.

27. G. Mishra, D. Singh, P. K. Yadawa, S. K. Verma, R. R. Yadav, Study of Copper/ Palladium Nanoclusters Using Acoustic Particle Sizer, Plat Met Rev. 57 (2013) 186-192.

28. P. Rahimi, H. Hashemipour, M. Ehtesham Zadeh, S. Ghader, Experimental Investigation on the Synthesis and Size Control of Copper Nanoparticle via Chemical Reduction Method, Int. J. Nanosci. Nanotechnol. 6 (2010) 144-149.

29. H. Khmissi, A. El Sayed, M. Shaban, Structural, morphological, optical properties and wettability of spin-coated copper oxide; influences of film thickness, $\mathrm{Ni}$, and (La, Ni) co-doping, J.Mater. Sci. 51 (2016) 5924-5938. 


\section{Figures}

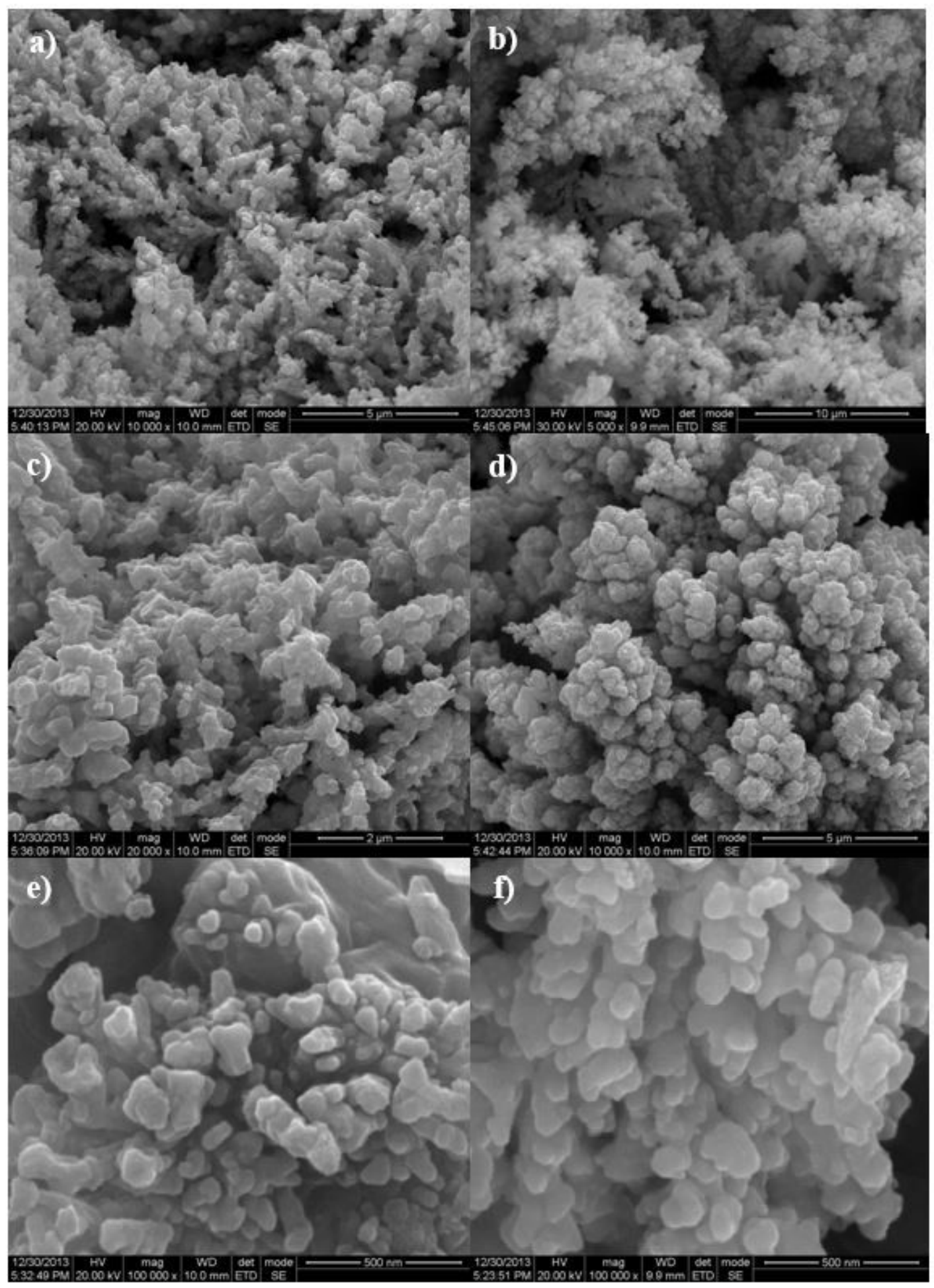

\section{Figure 1}

HR-SEM micrographs of CuZn bimetallic nanoparticles obtained at different reaction parameters (a-b) at a pH 4.5 with 0.02:0.01 mM concentration of $\mathrm{Cu}: \mathrm{Zn}$ at 20mA applied current, c) at a pH 5.5 with 0.02:0.02 
$\mathrm{mM}$ conc. at $20 \mathrm{~mA}, \mathrm{~d})$ at a $\mathrm{pH} 4$ with 0.02:0.02 $\mathrm{mM}$ at $20 \mathrm{~mA}, \mathrm{e}$ ) at a pH 3.5 with 0.01:0.01 mM at $20 \mathrm{~mA}$, f) at a $\mathrm{pH} 3.5$ with $0.01: 0.01 \mathrm{mM}$ at $10 \mathrm{~mA}$ (optimized condition)

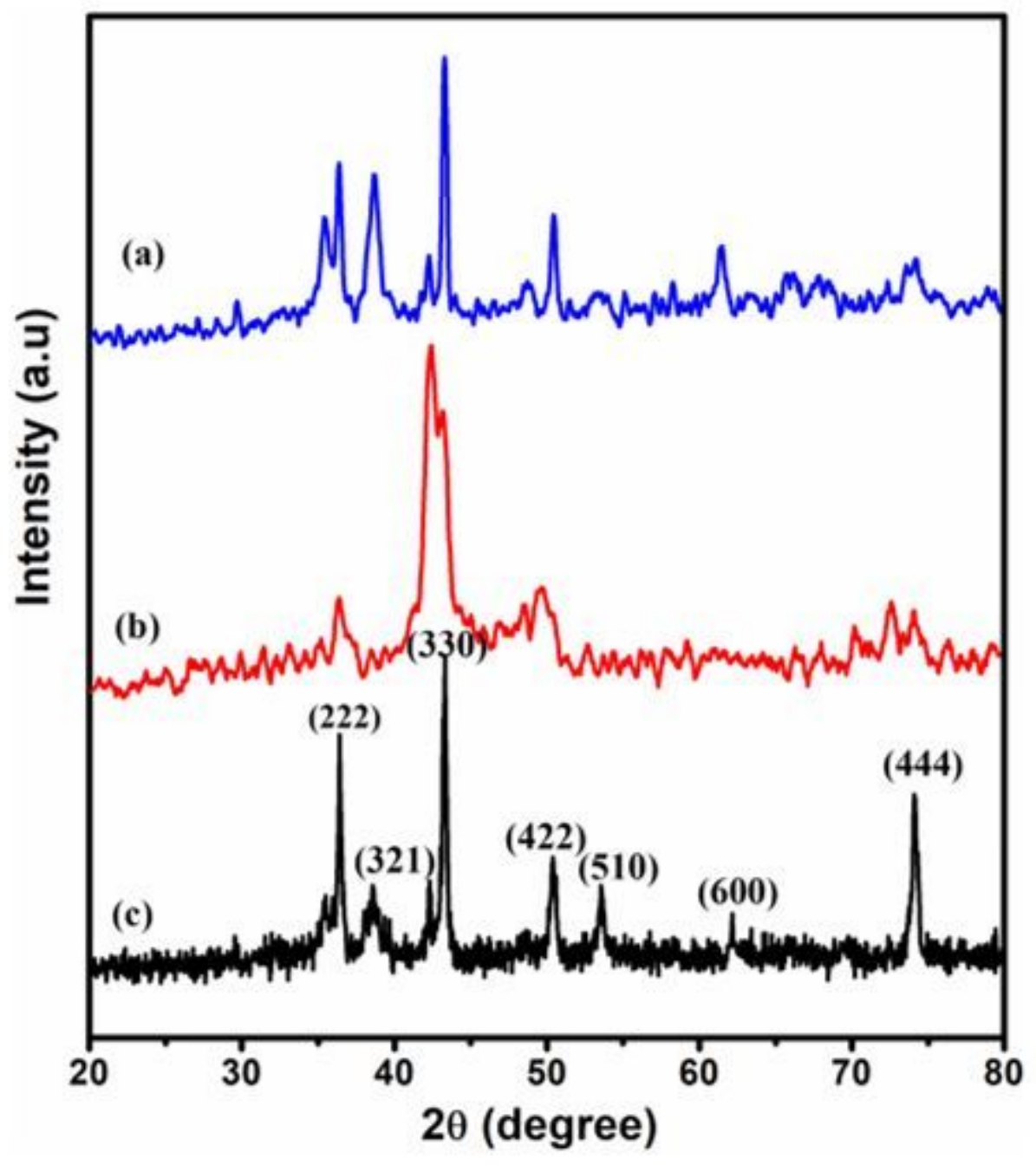

Figure 2

XRD patterns of CuZn bimetallic nano alloy electrodeposited on to the graphite electrode at different reaction conditions. A) at a $\mathrm{pH} 4.5$ with 0.02:0.01 mM concentration of $\mathrm{Cu}: \mathrm{Zn}$ at $20 \mathrm{~mA}$ applied current, B) at a pH 5.5 with 0.02:0.02 mM conc. at $20 \mathrm{~mA}$, e) at a pH 3.5 with 0.01:0.01 $\mathrm{mM}$ at $20 \mathrm{~mA}, \mathrm{C})$ optimized condition - at a $\mathrm{pH} 3.5$ with 0.01:0.01 $\mathrm{mM}$ at $10 \mathrm{~mA}$ 


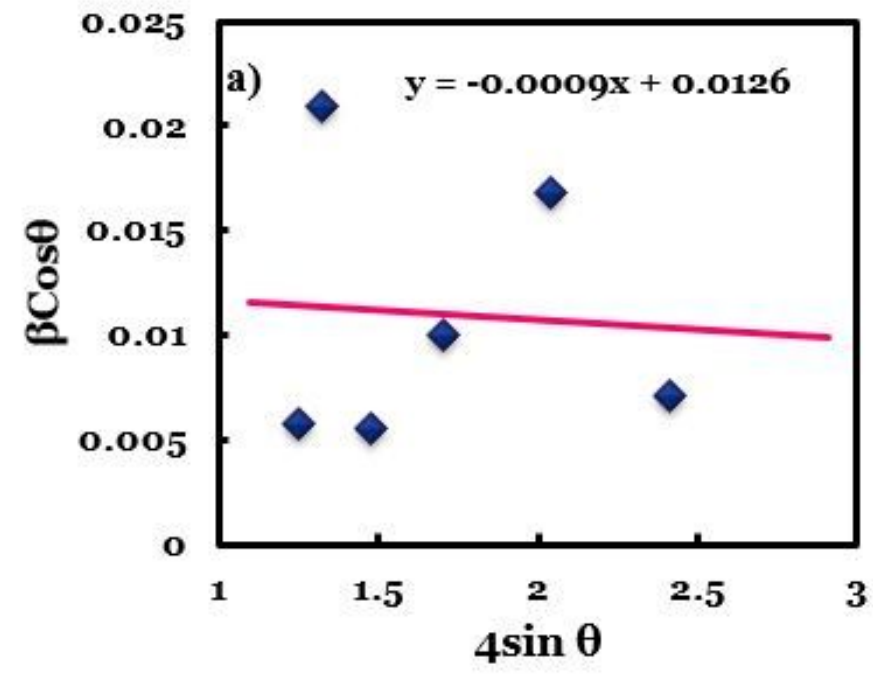

Slope $=-0.0009$ Particle size $=11.0128$ nm

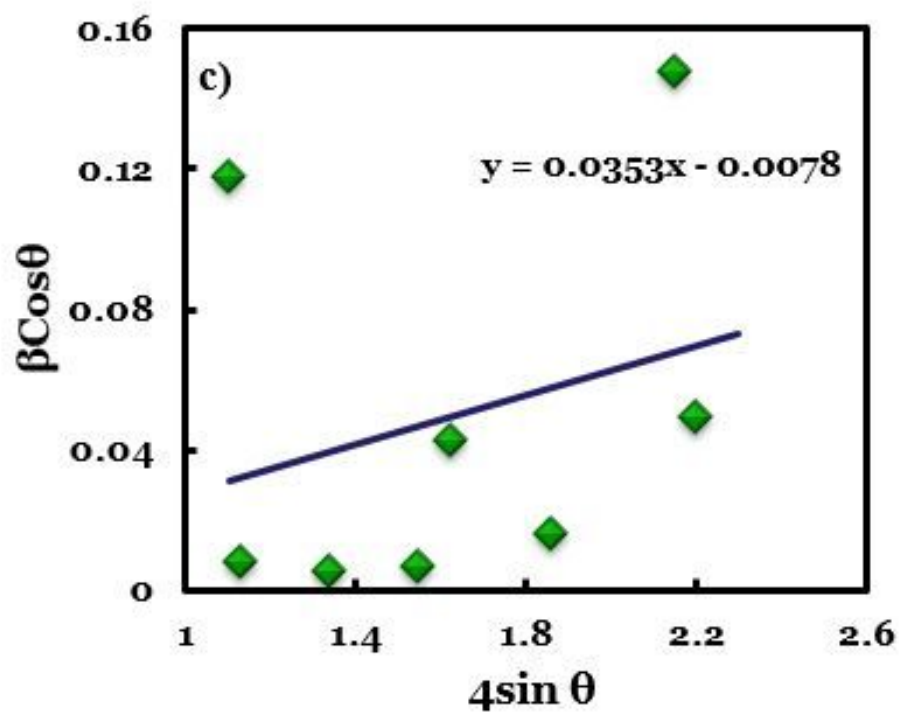

Slope $=0.0353$ Particle size $=0.0078 \mathrm{~nm}$

Figure 3

(a-c) Williamson-Hall plots of CuZn alloy

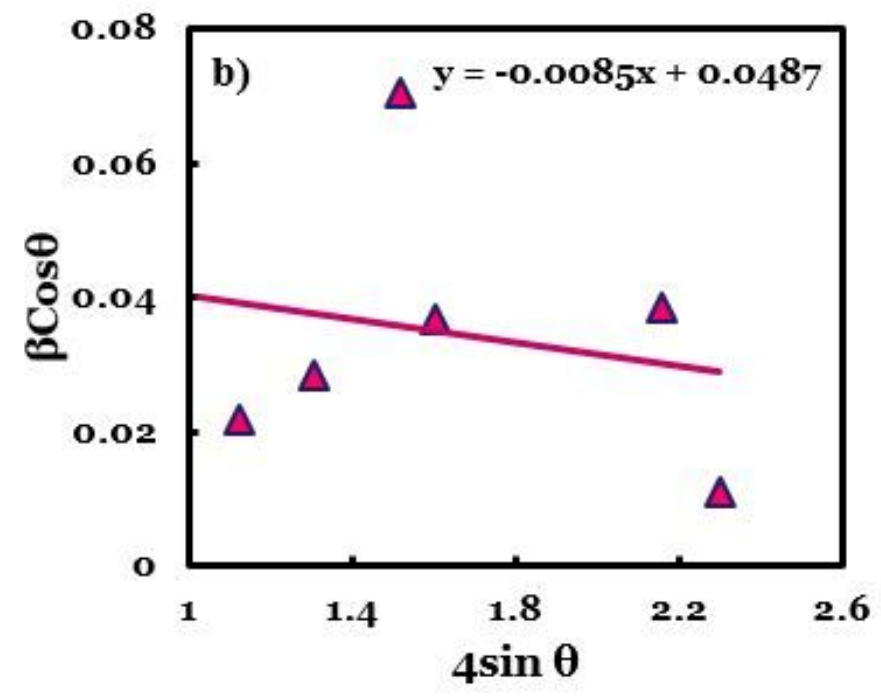

Slope $=-\mathbf{0 . 0 0 8 5}$ Particle size $=\mathbf{2 . 8 4 9 3} \mathrm{nm}$ 


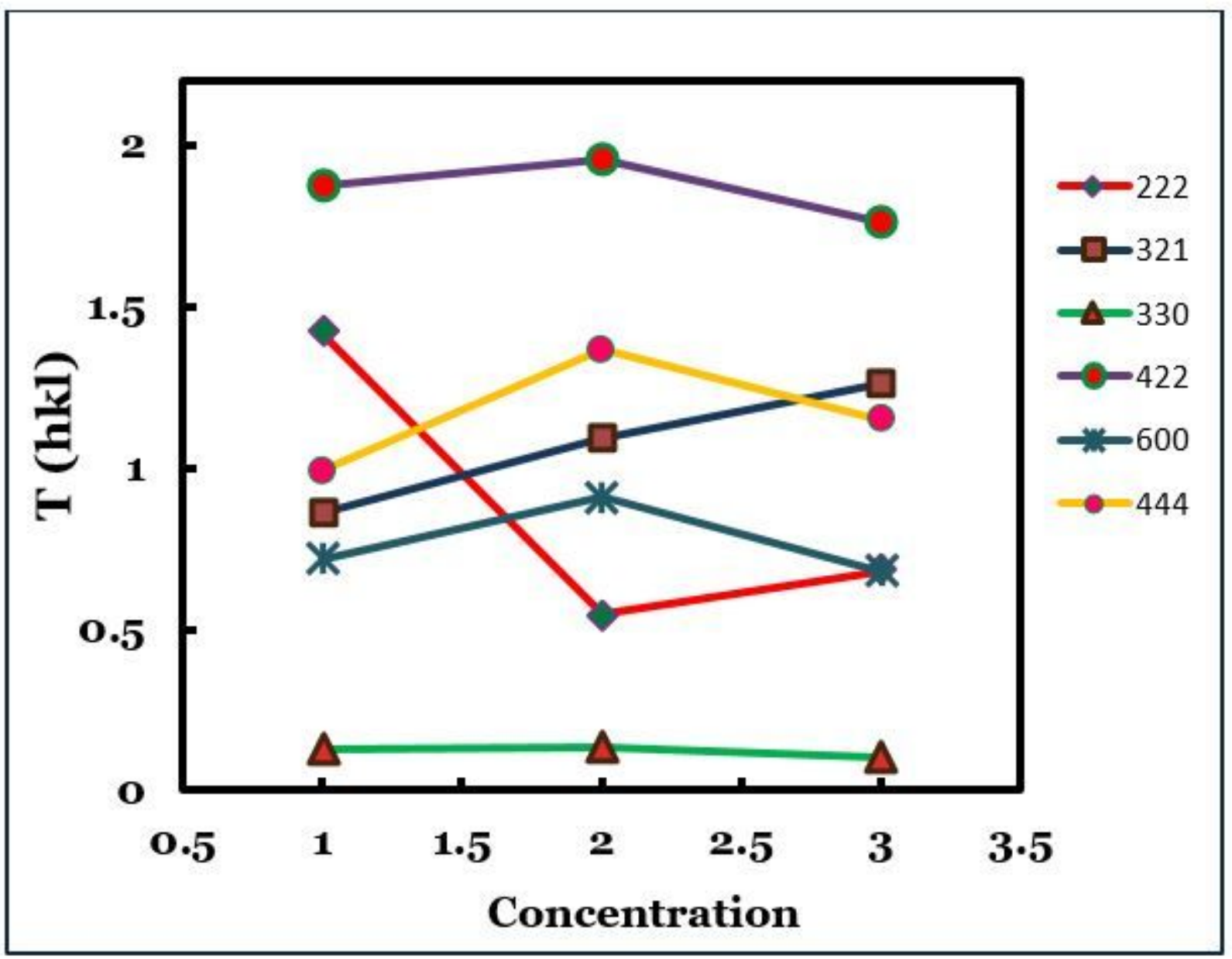

Figure 4

Texture coefficient T(hkl)_i of the CuZn bimetallic alloy obtained at different electrolytic concentration 

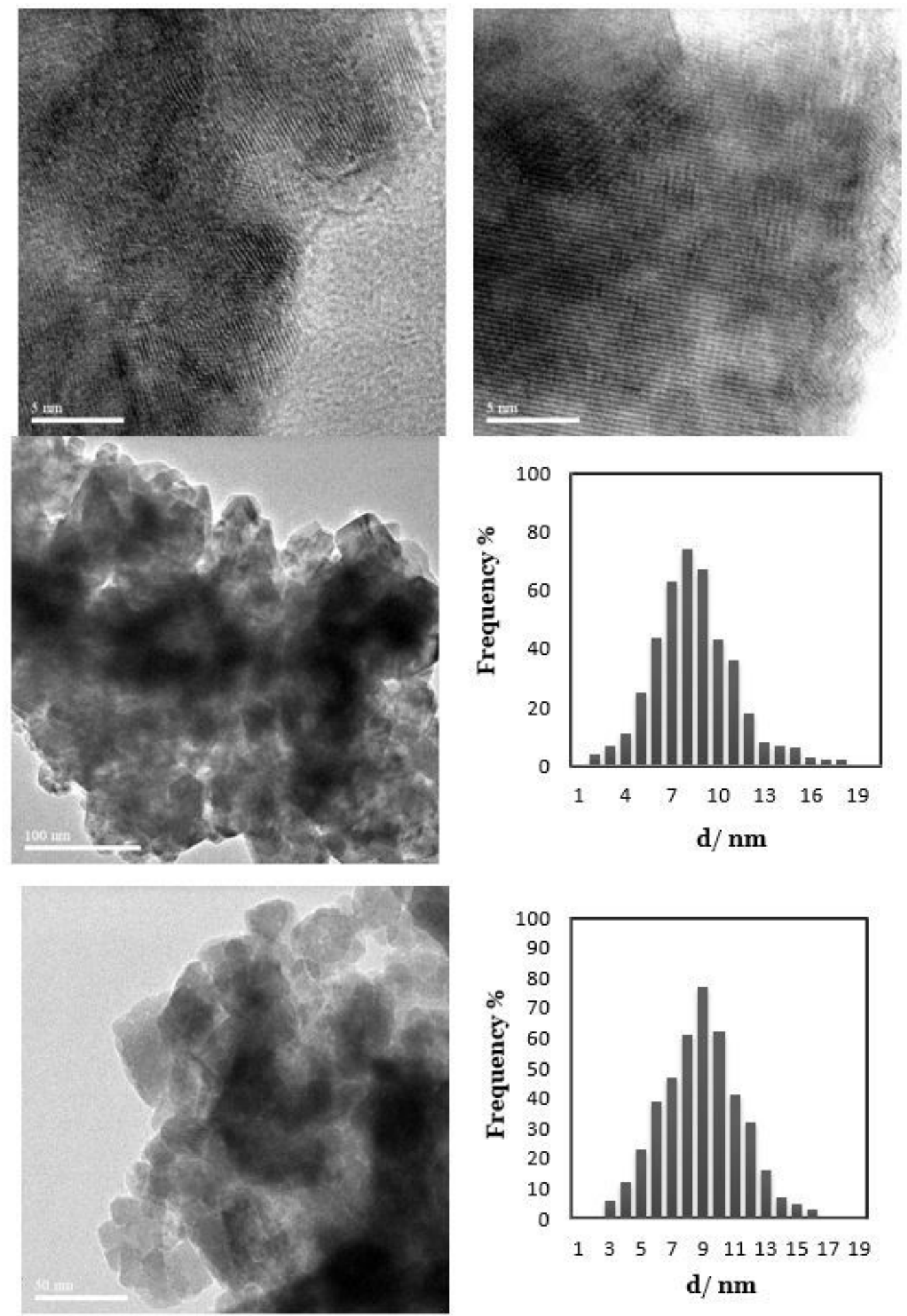

Figure 5

TEM micrographs and particle size histogram of CuZn nano alloy prepared from eletrodeposition method 

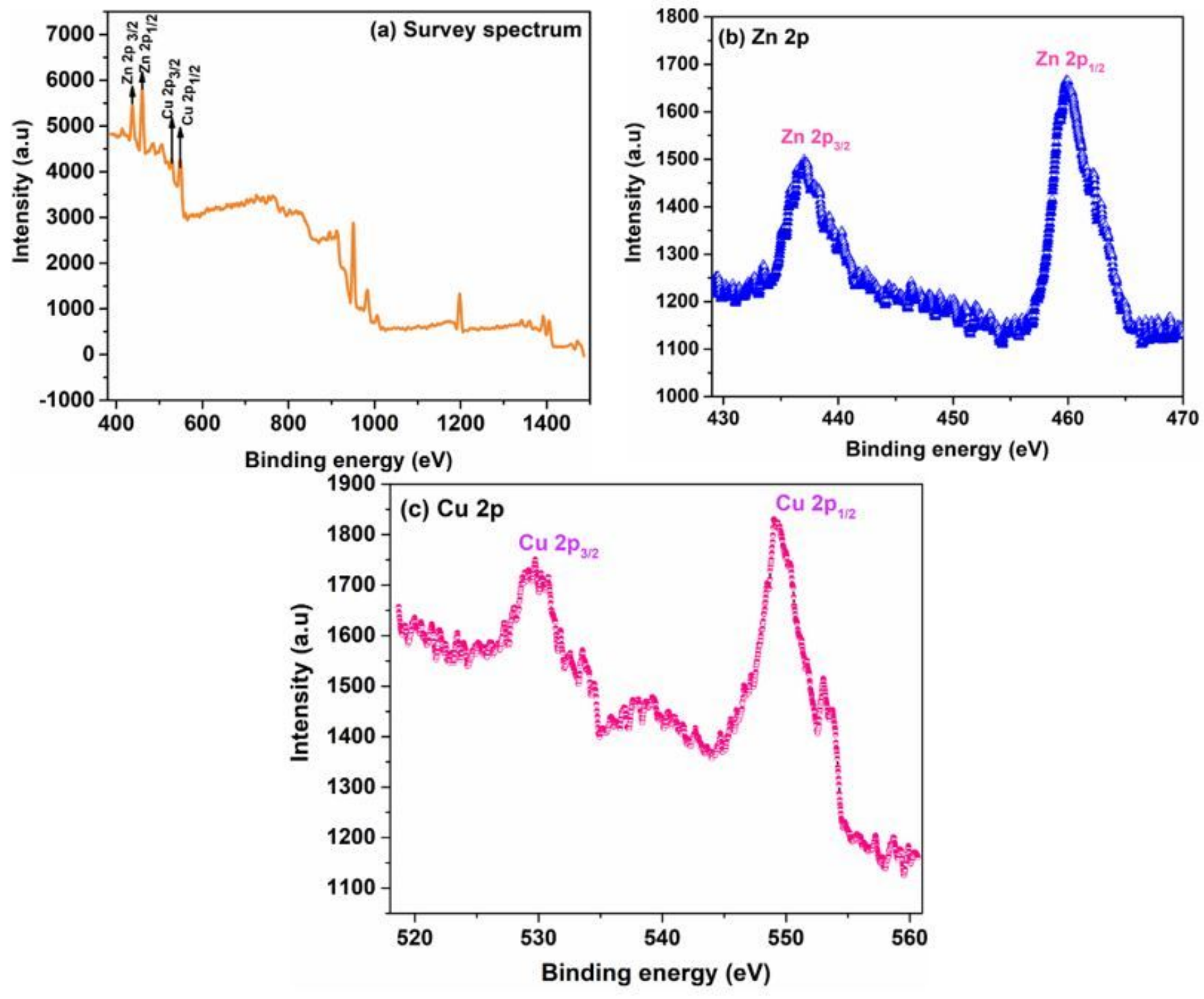

\section{Figure 6}

XPS analysis of CuZn alloy (a) survey spectrum of $\mathrm{y}$-Cu5Zn8 bimetallic nano alloy (b) high resolution XPS $2 p$ spectrum showing Zn 2p3/2 peak at 443 and $2 p 1 / 2$ peak at $465 \mathrm{eV}$, (c) spectrum for $\mathrm{Cu} 2 \mathrm{p} 3 / 2$ peak at 535 and $2 \mathrm{p} 1 / 2$ peak at $555 \mathrm{eV}$ 


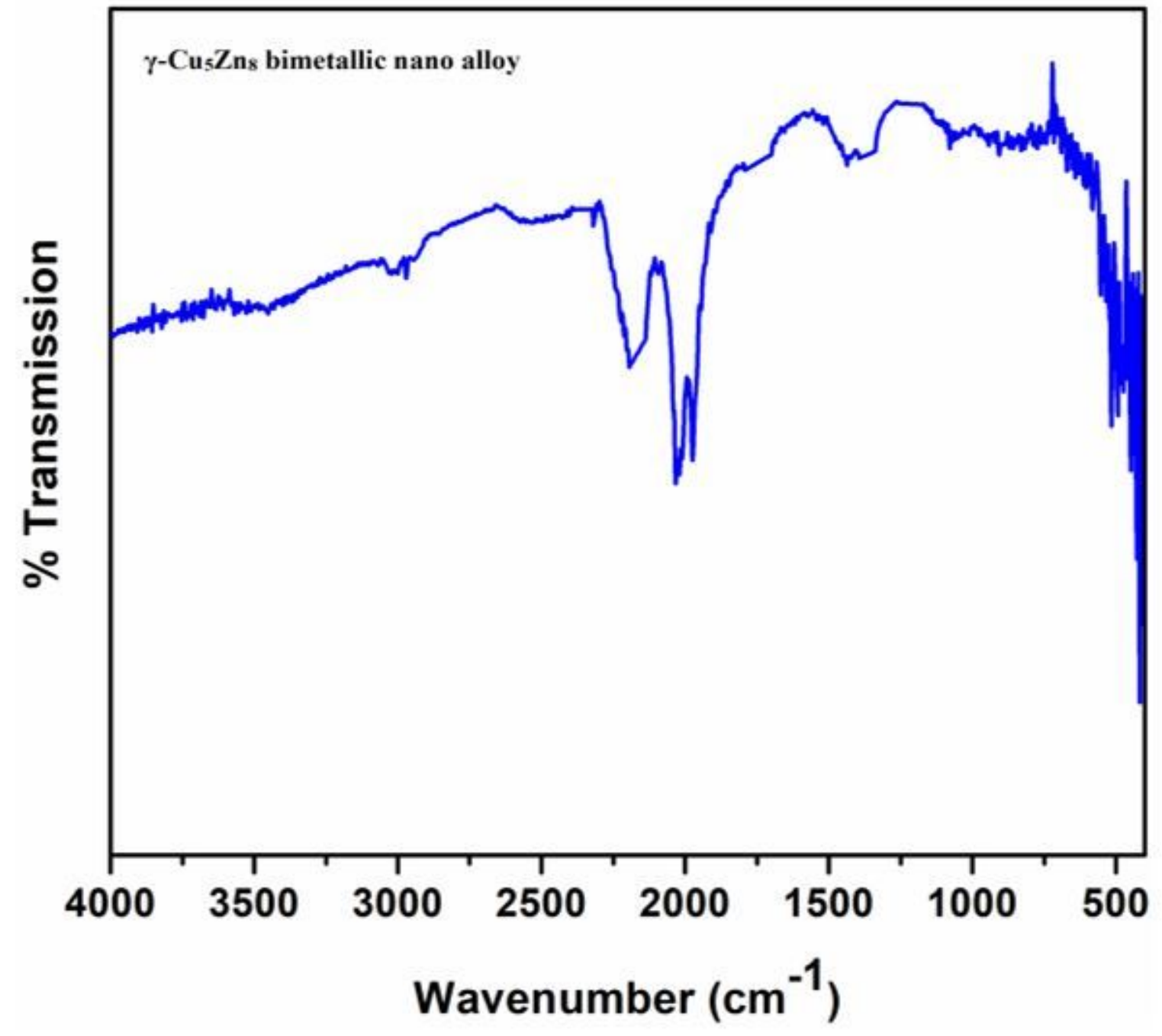

Figure 7

FT-IR spectrum of CuZn alloy 


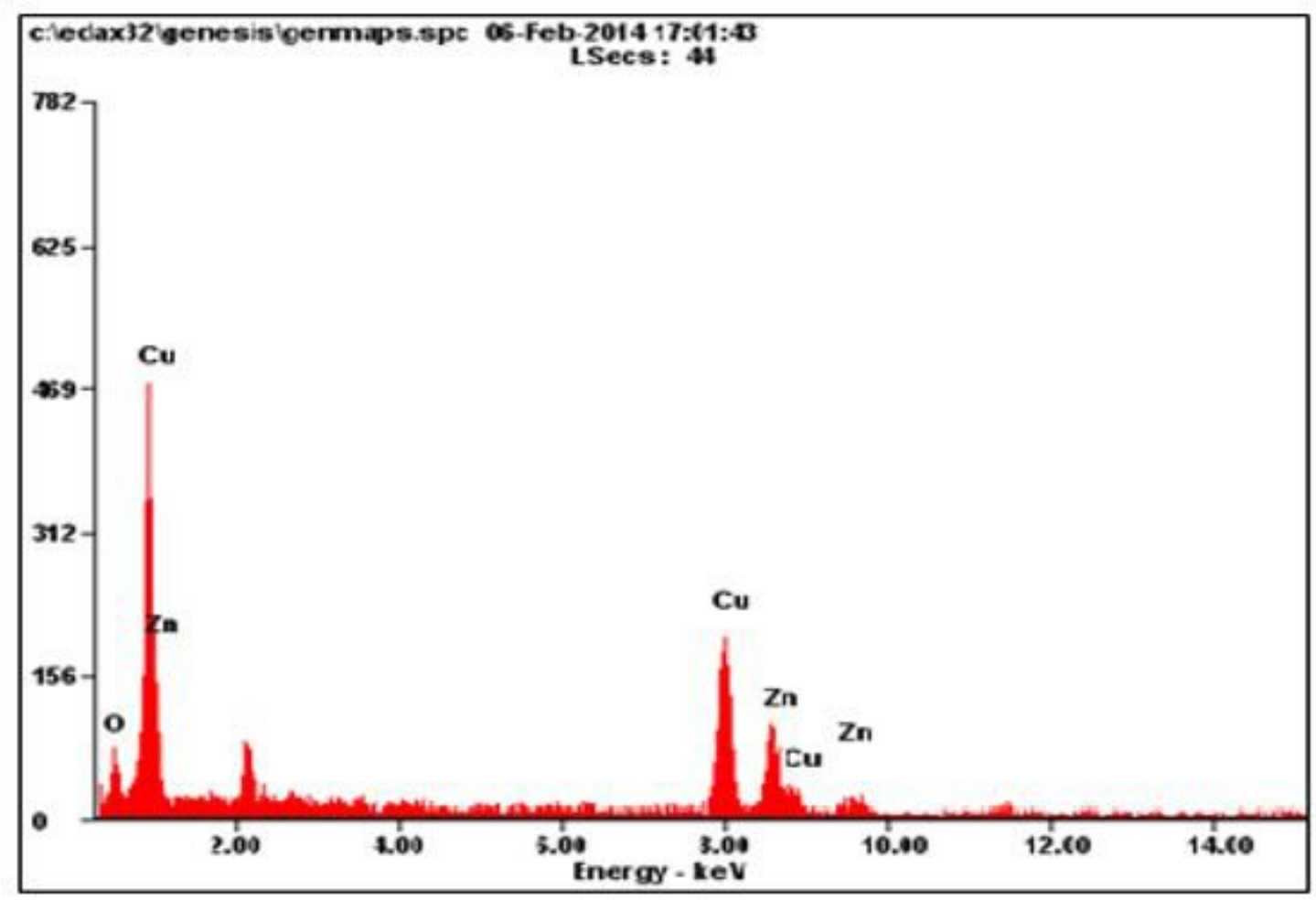

\begin{tabular}{||l|l|l||}
\hline Element & Wt\% & At\% \\
\hline $\mathrm{OK}$ & 5.42 & 18.25 \\
\hline $\mathrm{CuK}$ & 53.46 & 48.51 \\
\hline $\mathrm{ZnK}$ & 35.12 & 33.24 \\
\hline Matrix & Correction & ZAF \\
\hline \hline
\end{tabular}

Figure 8

EDX analysis of CuZn alloy 

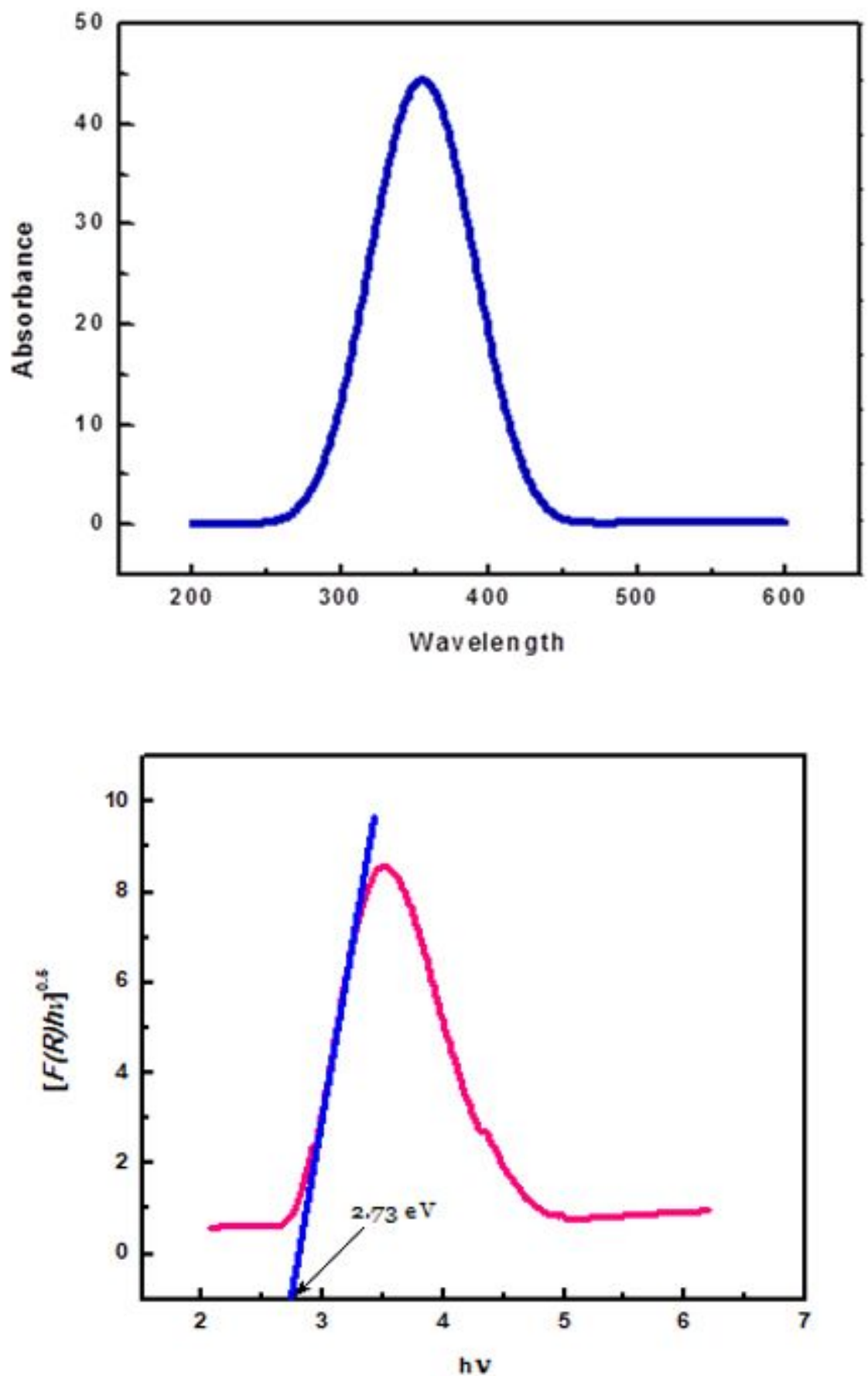

Figure 9

(a) UV-DRS spectrum of CuZn nano alloy (b) Kubelka-Munk plot of CuZn nano alloy showing band gap energy 

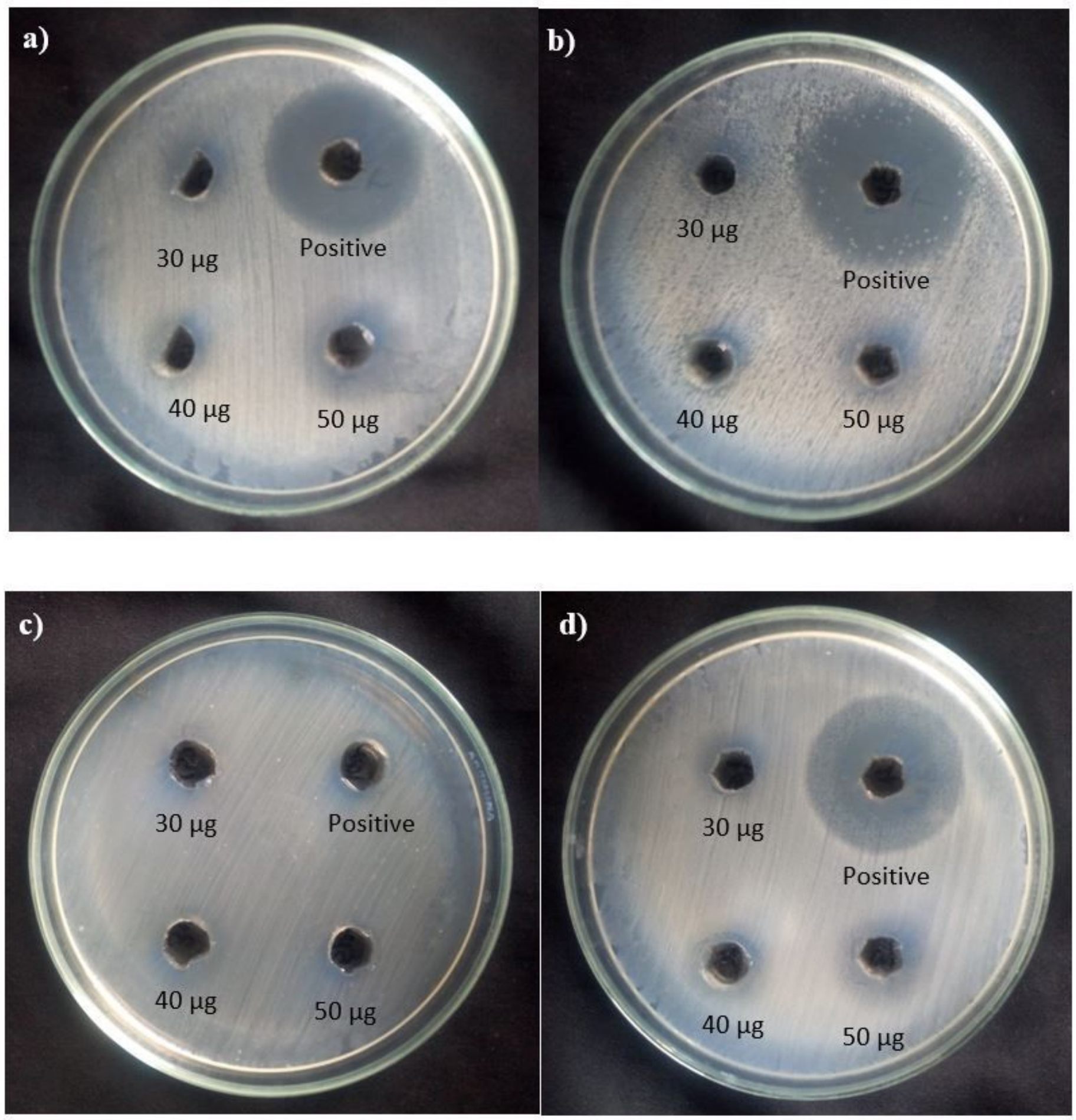

Figure 10

Well diffusion assay showing Zone of inhibition of CuZn alloy at optimum reaction conditions against a) Klebsiella pneumoniae b) Escherichia Coli, c) Bacillus Subtilis and d) Staphylococcus Aureus 

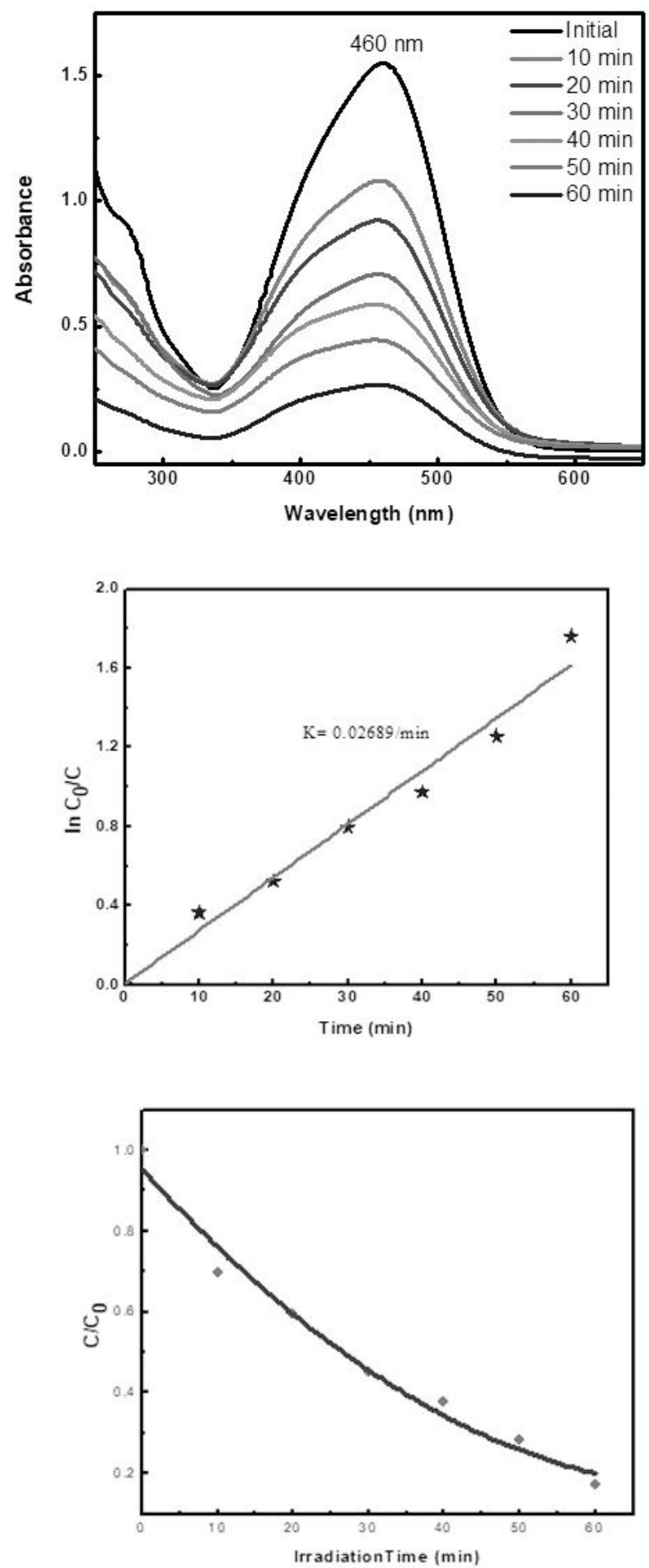

Figure 11

(a) UV-visible absorption spectra showing the degradation of Methyl Orange upon sunlight irradiation using $Y$-CuZn alloy (b) Pseudo first order kinetics for photocatalytic degradation of methyl orange dye using $\mathrm{Y}$-CuZn nanocatalyst (c) photocatalytic degradation profile of MO by CuZn nano alloy 